\title{
A New Green Prospective of Non-orthogonal Multiple Access (NOMA) for 5G
}

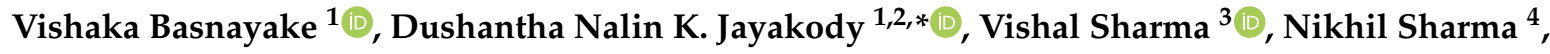 \\ P. Muthuchidambaranathan ${ }^{5}$ and Hakim Mabed ${ }^{6}$ \\ 1 Centre for Telecommunication Research, Faculty of Engineering, Sri Lanka Technological Campus, \\ Padukka 11500, Sri Lanka; vishakab@sltc.ac.lk \\ 2 School of Computer Science and Robotics, Research Tomsk Polytechnic University, Tomsk 634050, Russia \\ 3 ISTD Pillar, Singapore University of Technology and Design (SUTD), Singapore 487372, Singapore; \\ vishal_sharma2012@hotmail.com \\ 4 ECE Department, The LNM Institute of Information Technology, Jaipur 302031, India; \\ nikhilsharma.nit90@gmail.com \\ 5 ECE Department, National Institute of Technology Tiruchirappalli, 620015 Tamilnadu, India; \\ muthuc@nitt.edu \\ 6 DISC/FEMTO-ST, University Bourgogne Franche-Comte, 25200 Montbeliard, France; hmabed@gmail.com \\ * Correspondence: nalin@tpu.ru
}

Received: 31 December 2019; Accepted: 4 February 2020; Published: 7 February 2020

\begin{abstract}
Energy efficiency is a major concern in the emerging mobile cellular wireless networks since massive connectivity is to be expected with high energy requirements from the network operators. Non-orthogonal multiple access (NOMA) being the frontier multiple access scheme for $5 \mathrm{G}$, there exists numerous research attempts on enhancing the energy efficiency of NOMA enabled wireless networks while maintaining its outstanding performance metrics such as high throughput, data rates and capacity maximized optimally.The concept of green NOMA is introduced in a generalized manner to identify the energy efficient NOMA schemes. These schemes will result in an optimal scenario in which the energy generated for communication is managed sustainably. Hence, the effect on the environment, economy, living beings, etc is minimized. The recent research developments are classified for a better understanding of areas which are lacking attention and needs further improvement. Also, the performance comparison of energy efficient, NOMA schemes against conventional NOMA is presented. Finally, challenges and emerging research trends, for energy efficient NOMA are discussed.
\end{abstract}

Keywords: energy efficiency; energy harvesting; green communication; interference cancellation; non orthogonal multiple access; optimization; simultaneous wireless information and power transfer; $5 \mathrm{G}$

\section{Introduction}

In parallel to providing the expected connectivity of 5G, it is expected that the 5G network will be a more energy efficient, self-sustained, low budget, globally available to all resource, than the existing LTE networks. It is predicted that the mobile traffic in the emerging 2020 era will be 1000 times more and there will be 100 billion devices connected which will pose a huge challenge for the mobile network industry [1-4]. Moreover, as users are looking for a cost-effective high data rate, high capacity network, there are many research contributions towards enhancing the performance of the 5G mobile network. With the expected high number of end terminals or devices and applications requested from $5 \mathrm{G}$, the multiple access scheme has a significant contribution. In this regard, non-orthogonal multiple access (NOMA) is a flexible communications technology that can cater for a massive number 
of simultaneous connections with the available bandwidth [5]. Therefore, NOMA scheme has been recently proposed as the most optimal multiple access strategy for the current 3GPP LTE and the upcoming 5G mobile networks [6-8]. One of the most significant contribution of NOMA is the enhanced spectral efficiency, thus it can serve multiple users in the same time, frequency or with the same code but with different power allocations. Hence, yielding a significant spectral efficiency compared to the conventional orthogonal frequency division multiple access (OFDMA). Furthermore, NOMA scheme can be combined with many other technologies to cater the requirements of high spectral efficiency, very low latency, massive device connectivity, very high achievable data rate, ultra-high reliability, excellent user fairness, high throughput, supporting diverse quality of services (QoS), energy efficiency and reduction of operating costs expected from 5G networks [9]. Thus, NOMA incorporated technologies such as MIMO-NOMA, cooperative NOMA, NOMA, and cognitive radio (CR) and green NOMA have attracted much attention from the research community [10].

Energy-efficient NOMA or green NOMA will be a key ingredient in the upcoming 5G mobile network design to cater for the goal of achieving a low cost, self-sustaining, environment-friendly wireless network. It has been observed from the Global e-Sustainability Initiative that in 2002, the mobile industry was producing around $43 \%$ of the global information and communications energy-related emissions and it is expected that this will rise to $51 \%$ by 2020 [11]. With the ever increasing humans to human and machine to machine communications and the advent of the concepts of smart cities, smart homes and smart transportation, the future networks must be designed to achieve up to 1000 times the capacity of currently available networks. Catering high end mobile service requirements will result in a huge carbon print. Therefore, sustainable energy-saving techniques create a big impact on reducing the effect on the environment as well as the economy. The carbon emissions generated by mobile devices and the operation of mobile radio access networks will remain major contributors to the global carbon footprint due to the rising mobile traffic volumes in the years to come [12]. Therefore, maintaining energy efficiency and sustainability in the design and operation of wireless communication systems is a major concern. For more than a century, attention was mostly driven towards optimizing performance metrics such as the data-rate, throughput, latency, etc. However, in the last decade, energy efficiency has emerged as a new prominent figure of merit due to economic, operational and environmental concerns. Also, more than $80 \%$ of a typical mobile network operator's energy requirements are associated with operating the network [11].

Hence, since NOMA is the multiple access technology proposed for 5G network requirements, the design of NOMA networks will necessarily have to consider energy efficiency as one of its key performance indicators. Thus, the concept of green NOMA is introduced in a generalized manner to identify the energy efficient NOMA schemes. These schemes will result in an optimal scenario in which the energy generated for communication is managed sustainably. Hence, the effect on the environment, economy, living beings, etc is minimized. It is observed in [13] how the distributed energy production from renewable energy sources can generate sufficient electrical energy and also avoid carbon dioxide emission. Figure 1 illustrates the major existing energy efficient technologies and their applied scenarios in wireless mobile-cellular networks.

Mathematically, energy efficiency is defined as the amount of information that can be reliably transmitted per Joule of consumed energy; which is denoted using units of bits-per-Joule [14]. Even though many research works proposed many solutions for energy efficient NOMA, however the output appears to be insufficient to face the high energy consumption issue of 5G networks [15]. This study aims to discuss the beneficial role of energy harvesting technology in $5 \mathrm{G}$ networks. We categorize and classify the literature Table 1 available on energy efficiency for NOMA based networks. The key requirements and challenges for enabling energy efficiency networks are also outlined.

Our contributions in this article are:

- Extensive classification of research works on NOMA related energy efficient technologies under power and code domain. 
- Proposition of strategies for extending the energy efficiency of existing green NOMA schemes incorporating state-of-the-art techniques.

- Identifying challenges and future directions for energy efficient NOMA.

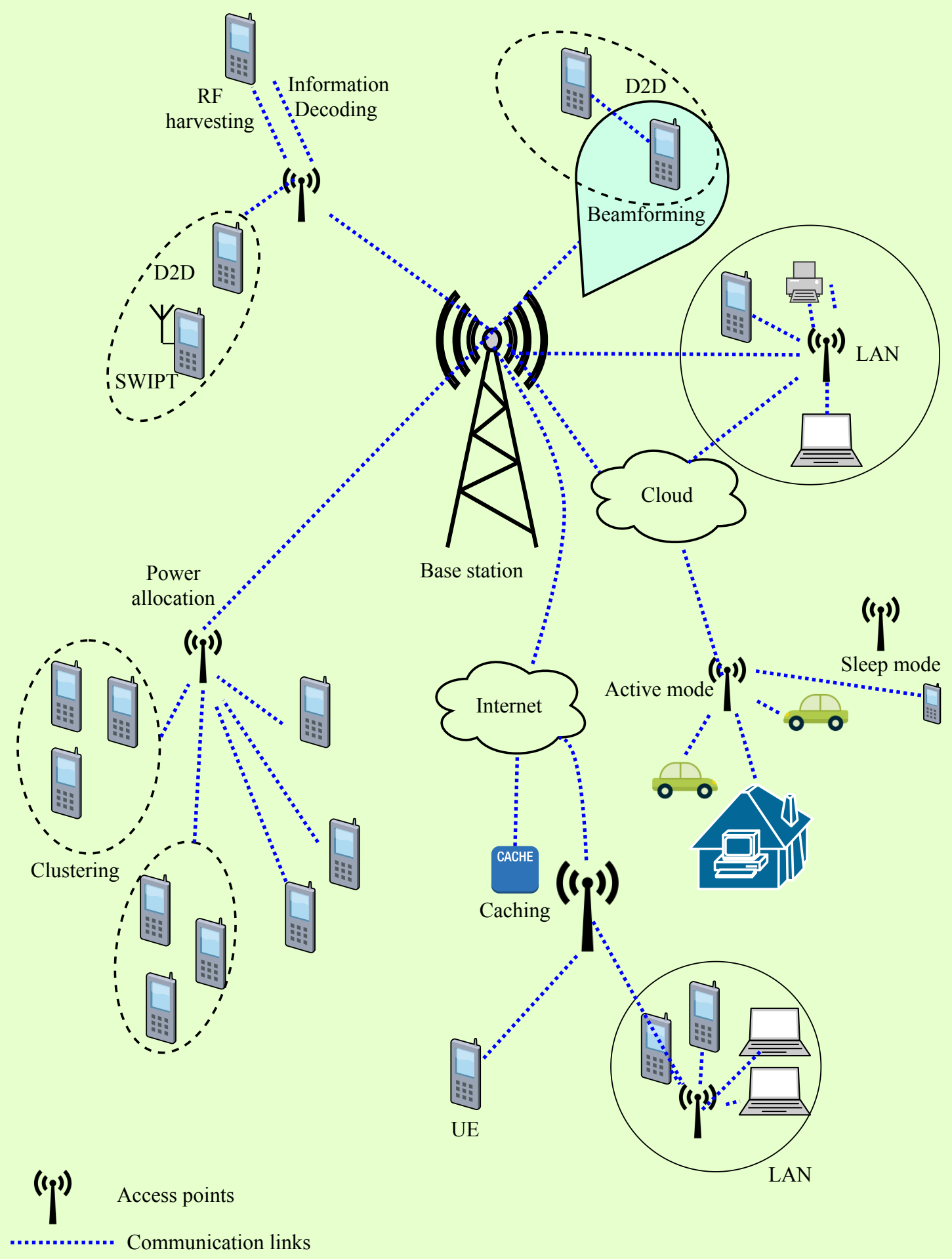

Figure 1. Existing schemes of enhancing energy efficiency in NOMA based wireless networks such as power allocation, RF energy harvesting, cooperative communications (D2D, M2M), cloud, caching, beamforming, sleep/active modes. 
Table 1. Classification of research work related to energy efficient NOMA which are discussed in this article.

\begin{tabular}{clc}
\hline Domain & Technology & Citations \\
\hline \multirow{2}{*}{ Power domain } & Resource allocation & {$[16-35]$} \\
& Energy harvesting and transfer & {$[36-49]$} \\
& Interference cancellation techniques & {$[50-55]$} \\
& Sleep/wake modes & {$[56-60]$} \\
Code domain & Sparse code multiple access (SCMA) & {$[61,62]$} \\
& Space time block coding (STBC) & {$[56,63]$} \\
& Polar codes & {$[64-66]$} \\
& Low density parity check (LDPC) & {$[67-69]$} \\
& Multi user shared access (MUSA) & {$[70,71]$} \\
\hline
\end{tabular}

Additionally, the basic principles of NOMA and energy efficient NOMA are also discussed and the recent research works and challenges incorporating green concept are revisited and the possible future directions are presented. Hence, in-depth the recent state of art for energy efficiency for NOMA based networks are presented in the proceeding section.

\section{Classification of Green NOMA Technologies}

Facing massive connections and unavoidable interference, the ways of providing green communication for emerging mobile networks is an important matter. Thus, it is our goal to review and investigate available strategies in maintaining and increasing the energy efficiency of all users in a NOMA network. Hence, related research works on enhancing energy efficiency are revisited and presented in this paper. In most of the existing literature [16-27], proposed algorithms are derived using probability, optimization, signal processing principles and are used to derive optimal or sub-optimal energy efficient algorithms. The approaches for green NOMA of wireless networks can be classified under two major categories namely power domain and code domain NOMA as illustrated in Figure 2. Further, all the proposed methods for green NOMA are compared with the prevailing multiple access schemes in the current 3GPP mobile networks and their performances evaluated.

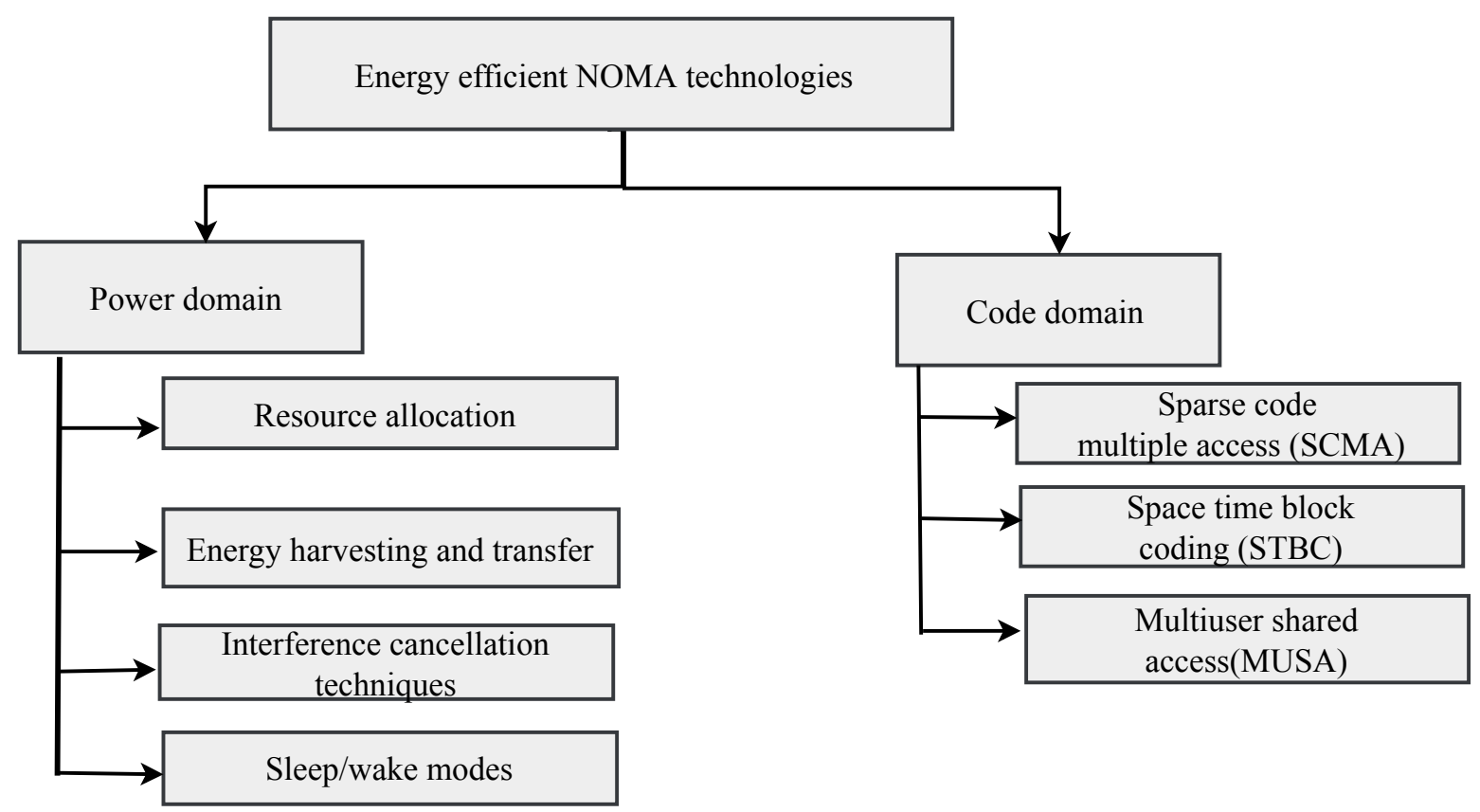

Figure 2. Classification of green technologies for NOMA based on existing research work discussed in this article. 


\section{Power Domain based NOMA}

\subsection{Resource Allocation}

A lot of literature discussed on the resource allocation to achieve energy efficiency of NOMA [16,18-28]. Resource allocation is a key area to achieve the full potential of communication systems with NOMA [19]. With the notion of achieving energy efficiency in NOMA systems under resource allocation, the research contribution can mainly be classified under power allocation, channel assignment, transmission scheduling, user selection and joint schemes of these techniques. The contributions of research work under these categories are elaborated in the subsequent subsections. Also, the choice of which resource allocation to use depends on the type of communication system that will be applied. Therefore, it is necessary to choose the optimal strategy according to the specific scenarios.

\subsubsection{Power Allocation}

Power allocation strategies play a major role under resource allocation and thus, the techniques used in the current research work on power allocation and optimization for energy efficiency are addressed here. Besides, Figure 3 provides an overview of power allocation technologies and related fields that enhance the energy efficiency of NOMA based wireless networks.

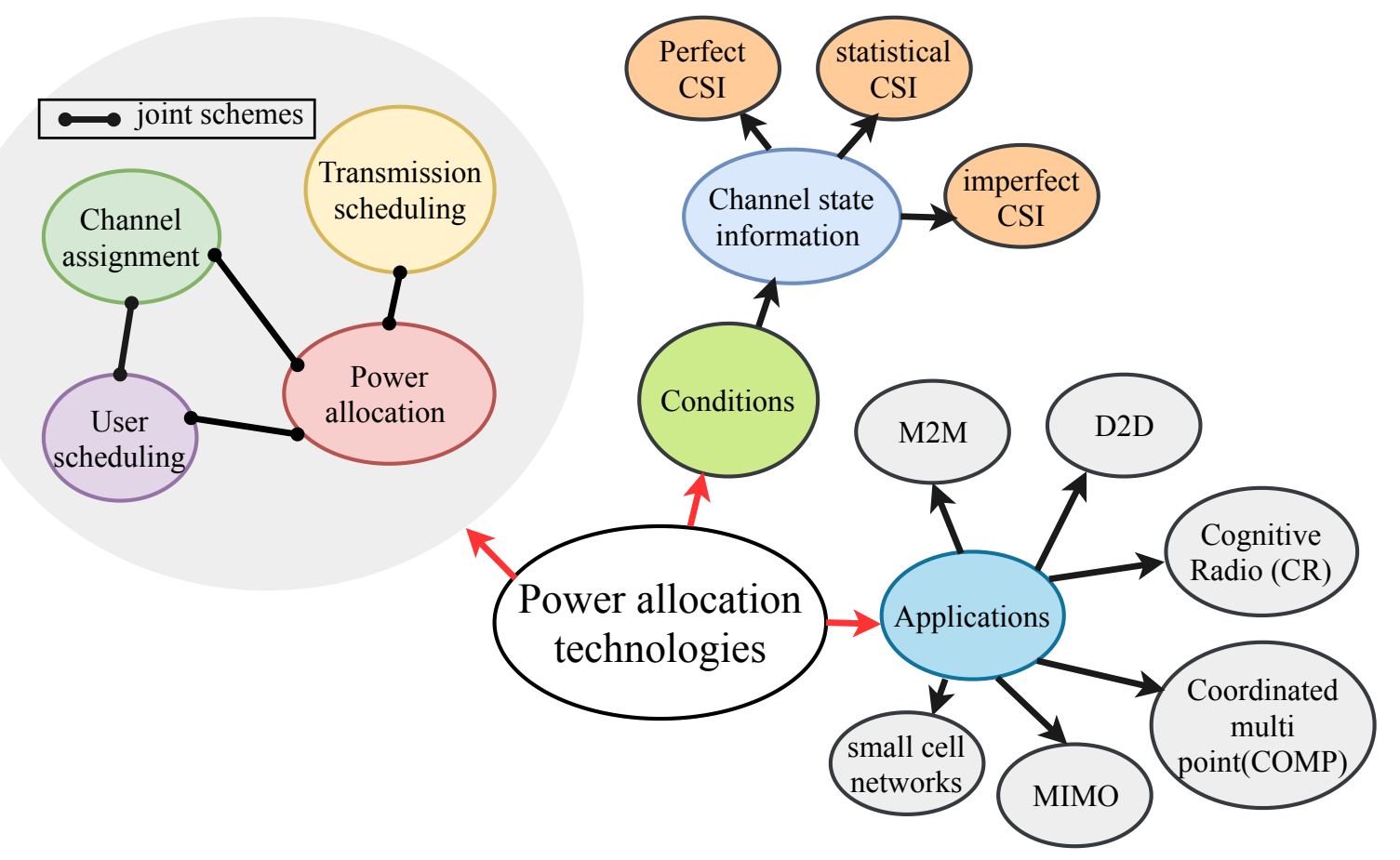

Figure 3. Classification of energy efficient green technologies for NOMA under power allocation scheme.

First, the paper [21] addresses an adaptive power minimization strategy which utilizes an optimization algorithm to minimize power allocation and determine the optimal user equipments (UEs) grouping for a certain subchannel. The number and the composition of the UEs group are also derived. Therefore, both power allocation and UEs grouping are optimized here to obtain the best performance of the NOMA scheme. In [17], an optimal energy efficient power allocation scheme is proposed for a NOMA system, where multiple users have their own data rate requirements. Further, the minimum required transmission power to satisfy the total data rate of all users is computed. Moreover, the numerical results of the optimal energy efficient power allocation scheme outperforms the energy efficiency performance of conventional orthogonal multiple access (OMA) systems. This 
is due to the fact that NOMA scheme can serve multiple users simultaneously via power domain division which makes it more energy efficient.

In $[16,18,20,22-24]$, derivation of optimal power allocation strategies under different categories of channel state information (CSI) namely, known CSI, statistical CSI and imperfect CSI have been studied to improve the performance in terms of providing minimum per user data rate requirements and minimization of transmission power. In $[18,20]$, energy efficient allocation algorithms were proposed for single-cell downlink NOMA with perfect CSI. Here, a low complex optimization algorithm is presented for subchannel assignment and power allocation for subchannel users. Also, it is revealed that the proposed algorithms receive a considerable enhancement in the sum rate and energy efficiency compared with the conventional OFDMA scheme. In [25], the authors have focused on the energy efficiency optimization for fading MIMO NOMA systems with statistical CSI at the transmitter. Here, the energy efficiency maximization problem in fading MIMO channels, under both the total transmission power constraint and the minimum transmission rate constraint of the weaker or farther user is addressed. In [22], an optimal power allocation solution that minimizes the total transmission power with throughput constraints for downlink NOMA under statistical CSI is presented. Using this, NOMA can guarantee certain throughputs for users with the minimum total transmission power when only statistical CSI is available at the base station (BS).

Research work, in [23], investigates the NOMA system under imperfect CSI and proposes two power allocation algorithms. First, a power allocation algorithm that can be employed in both uplink and downlink NOMA systems is proposed. The proposed algorithm is shown to provide the optimal solution to the rate maximization problem without relying on perfect CSI. Secondly, the earlier proposed algorithm is extended to consider various rate requirements of users. The extended algorithm considers the heterogeneous rate requirements of users and provides solutions based on a novel rate measure scheme. In [24], the authors consider the problem of power allocation for energy efficiency maximization in NOMA systems with imperfect CSI for a set of users. The total transmit power level is optimized under per user minimum rate requirement constraint.

In [26], the authors discuss a power allocation and channel assignment respectively. Here, the power allocation is optimized under multiple constraints using Lyapunov optimization. Moreover, it develops a subchannel assignment algorithm based on matching theory. In [27], the authors address a power efficient resource allocation algorithm with imperfect CSI for a multi-carrier NOMA (MC-NOMA) system. Also, the algorithm develops an optimal user scheduling based on the bottom-up approach of hierarchical clustering.

\subsubsection{Joint Schemes}

Moreover, the works [16,29-35] propose energy minimization solutions by jointly utilizing power allocation, user scheduling, transmission scheduling and channel assignment. Further, it is observed that the joint schemes are better than the single technique alone in terms of energy efficiency. In [29], the authors propose a joint transmission scheduling and power allocation algorithm to address the energy efficiency maximization problem. Here, they use practical data rate evaluation considering the modulation and coding compared to the analytical information-theoretic approach. In [30], authors resolve an energy minimization problem for uplink with a machine to machine system and NOMA based wireless network. Further, in [16] an energy efficient subchannel allocation algorithm was proposed for simple downlink NOMA with imperfect CSI. Here, an energy efficient optimization problem is formulated which combines user scheduling and power allocation schemes. It should be noted that channel estimation plays a major role in the performance of the algorithm. High channel estimation error deteriorates the performance of the proposed algorithm. Nevertheless, it is shown that the impact of the proposed resource allocation scheme in enhancing the energy efficiency of a NOMA system is better than in conventional OFDMA system. In [31], the problem of energy efficiency optimization is addressed for downlink NOMA by joint power allocation and channel assignment strategies. Here an optimal subchannel matching scheme is provided for the NOMA system using a 
game-theoretic approach. Thus, for a proposed subchannel matching, the power allocation problem is solved.

Furthermore, an energy optimization problem is addressed using joint user scheduling and power allocation scheme by considering both perfect CSI and imperfect CSI in [32] for downlink NOMA heterogeneous networks. NOMA heterogeneous networks consist of a macrocell overlaid by several small cells, where each of the small cells utilize the NOMA scheme. In [33], a joint user scheduling and power allocation problem is formulated as a stochastic optimization problem. Further, the long-term power consumption of the whole system including the BS and all mobile devices is minimized using the optimization problem created. Moreover, a joint combination of user scheduling, channel assignment to optimize the energy efficiency is proposed in [34]. A joint subchannel assignment and power allocation optimization problem is formulated in [35] for downlink NOMA coordinated multi-point (CoMP) networks. Here, both the total network and cell edge user throughput are maximized.

\subsection{Radio Frequency (RF) Energy Harvesting}

It is observed from the recent literature [44,72-77] that with the advent of energy efficient techniques like energy harvesting, the resulting self-sustained networks are getting attention of the research community. Energy harvesting and transfer have recently taken the attention in the field of wireless communication networks as an energy efficient mechanism that leads to self-sustaining, cost-effective networks. Recently, under RF energy harvesting communications, many research works have been done because energy harvesting has the potential to provide a continuous supply of power to the nodes in the communication networks [78]. Mainly, the focus has been driven towards investigating optimal energy harvesting techniques, protocols, transmission schemes and security in wireless communication networks.

Uplink energy efficient internet of things (IoT) NOMA based network which uses RF energy harvesting technology is given in [36]. Here, an efficient NOMA system is proposed which elaborates on the effect of energy harvesting radius against the user equipment density. Further, this shows that with the increment in user equipment density, the energy harvesting range increases. This shows that the cooperative energy sharing enables more energy harvesting for the users. In [37], authors propose a new relay selection scheme for energy harvesting cooperative NOMA based network. The relays are assumed to have no embedded energy supply and rely only on the energy harvested from the signals broadcasted by the source for cooperative NOMA transmission. In [38], an energy harvesting relay which supports multiple source-destination pairs to communicate is presented. Further, this strategy enables the energy harvesting relay to distribute the energy optimally among the multiple users. Further, it addresses two centralized methods for power allocation. The first one is an auction-based power allocation strategy where multiple destinations compete with each other for the assistance of the relay. This method provides a convenient trade-off between complexity and system performance. Secondly, the effect of a centralized method of water filling for power allocation is investigated. It shows an optimal performance, however it has high complexity. Furthermore, non-cooperative individual transmission strategy is shown to be inefficient compared to a cooperative communication system which allocates sufficient energy to the users with weak channel conditions.

In [39], a power splitting mechanism is derived for a simultaneous wireless information and power transfer (SWIPT) enabled NOMA network using a game theory approach that computes power splitting ratios for all relays to achieve maximum achievable rates for each user. Here, non-cooperative games are proposed for three different network scenarios and each link is modeled as a strategic player who aims to maximize its achievable rate by choosing the dedicated relay's power splitting ratio such that a good network-wide performance can be achieved. This is specially effective for systems with low and medium interference. The authors of [40] address the challenge of enhancing the energy efficiency of a M2M communication network, where energy is limited. Here the uplink scenario is considered and energy efficiency enhancement is achieved by combining both power control and time scheduling schemes. 
Several energy harvesting techniques that can be utilized for wireless sensor networks are given in [79]. Here, the major techniques which can be utilized for energy harvesting are classified as cooperative communication $[41,80,81]$, energy harvesting and wireless charging $[76,82,83]$, radio optimization [84], modulation optimization [46,85,86], sleep/wake up mechanisms [87-89], multi path routing and data reduction techniques such as adaptive sampling, compression and network coding.

In the era of 5G communication, SWIPT technology could be fundamentally important for energy and information transmissions within numerous types of modern communications networks. It is an emerging research area in wireless networks in enhancing energy efficiency and the sustainability of the network [79]. The concept of simultaneous wireless information and power transfer was first addressed in [90] which led to many research works related to SWIPT [43,49]. The work of [90] deals with the fundamental trade-off between transmitting energy and information over a single noisy line simultaneously. Energy harvesting and wireless transfer is an emerging area in enhancing the energy efficiency of wireless networks with many open problems.

The objective in [44] is to achieve self-sustaining energy harvesting wireless networks utilizing energy cooperation and SWIPT. SWIPT and cooperative relay communication are combined in [43], where the relays are randomly scattered. Here, energy is harvested at the relays from the relay receptions and the relay transmissions are powered by that energy. Further, the reliability of the transmission and energy efficiency is enhanced by the proposed algorithm where energy delivery and information transfer are conducted simultaneously by a single source. This solution is more energy efficient than the systems where the energy delivery and information transfer are decoupled and conducted by separate sources. Moreover, an energy efficient method for downlink energy harvesting NOMA relaying network is proposed in [41] with a three-phase harvest-transmit-forward transmission protocol. Furthermore, in [42], authors discuss how to apply SWIPT in NOMA using cooperative relaying. Here, a cooperative relay protocol in which near users act as energy harvesting relays to help the far users. Also, the advantage of opportunistic node location selection for user selection is performing better in terms of high throughput and low outage probability compared to random user selection scheme. It is concluded that by proper selection of network parameters such as transmission rate and power splitting coefficient, the expected energy efficiency can be obtained so that the users do not have to use their own batteries to power the relay transmission. Another instance of combining SWIPT and cooperative relaying is addressed in [45]. Here, the cell-center user, which is a full duplex relay, is used to support a cell edge user. The optimal power splitting and beamforming vectors are computed by an optimization algorithm ensuring enhanced energy efficiency and minimum required target rate for cell edge user and successful decoding rate at the cell center user. Further, the proposed strategy is used for an imperfect CSI system and it was found that the proposed solution can improve energy efficiency compared to existing strategies.

Further, authors in [46] propose a modulation based NOMA scheme and observe its efficiency in terms of higher harvested energy. It is a SWIPT enabled modulation based NOMA (M-NOMA) which shows higher energy efficiency outperforming conventional NOMA. Also, it proposes a SWIPT scheme where channel response is estimated using pilot symbols which are energy signals superimposed on the information signals and where energy is harvested from pilot symbols. Authors claim that the joint energy harvesting schemes and data rate fairness beamforming with distributed power beacons aid in enhancing energy efficiency which is illustrated in Figure 4. 


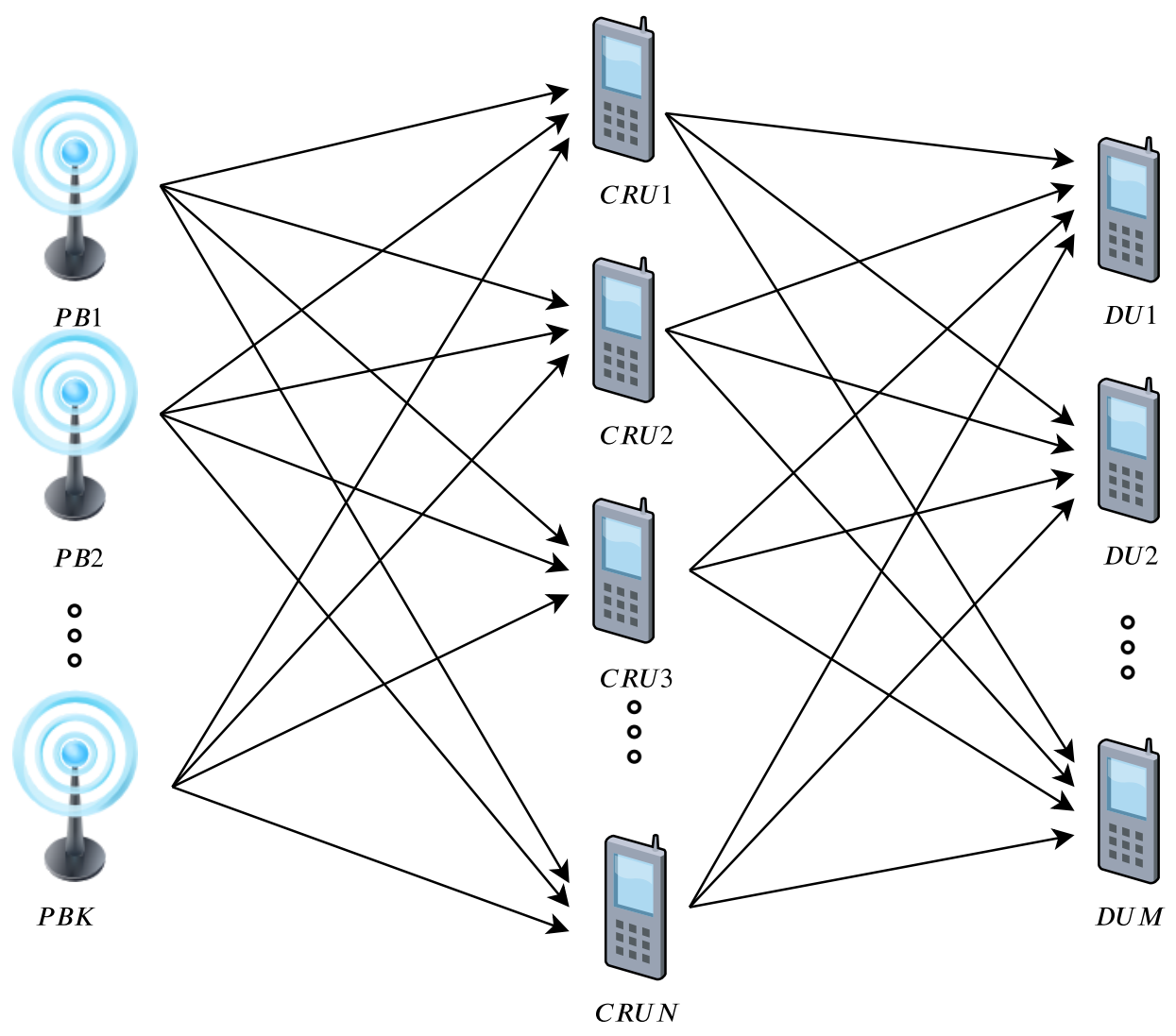

Figure 4. Proposed SWIPT scheme in [46] where cooperative relay users provide wireless power transfer and enable data rate fairness beamforming.

When the receiver architecture for SWIPT is considered, the authors propose two protocols for RF energy harvesting techniques in [47] as power splitting (PS) protocol and time switching (TS) protocol. The performance of these protocols are compared and the most energy efficient protocol is proposed. Hence, it is discovered that the time switching protocol is more energy efficient in lower transmission rates. In a higher transmission rate scenario, the information decoding cannot be done efficiently enough and thus the information transfer is not properly conducted. Therefore, the selection of the SWIPT protocol is a critical factor in conducting SWIPT of the wireless powered cooperative communication networks (WPCCNs). In [48], a power splitting protocol to enhance the performance of SWIPT assisted cooperative NOMA (SWIPT - CNOMA) system is unfolded. Here, the near users with strong channels act as energy harvesting relays that support the far users with weak channels. Furthermore, [49] addresses energy efficiency optimization using NOMA under combined power allocation and time switching techniques.

\subsection{Interference Mitigation and Cancellation}

The massive connectivity required in the emerging mobile networks for example in IoT scenarios results in unavoidable interference which is a huge challenge [5]. Mitigating interference is one of the major challenges in NOMA and recent research works on interference cancellation is detailed herewith. In [50] a method of minimizing interference between individual cells using coordinated approach between the cells and improving user throughput is explained. Research works describe another approach on interference cancellation, named as triangular successive interference cancellation (T-SIC) [51]. In this approach, multiple signals from each interfering user are collected and processed to create interference cancellation triangles. The strong users' symbol is detected before detecting the weak users' symbol and possible interference from the strong user is mitigated. In addition, interference cancellation using practical successive interference cancellation techniques are addressed 
in [52]. In this research work, authors study the effect of zero forcing (ZF) and minimum mean square error (MMSE) on interference cancellation in NOMA. Further, they modify ZF and MMSE schemes and develop a scheme which shows better interference cancellation than ZF and MMSE in a NOMA network. Further, in [53] an interference cancellation method namely, multiple interference cancellation (MIC) is introduced for a network with D2D pairs. MIC has a higher performance than the successive interference cancellation utilized in the conventional NOMA scheme. Here, the network coverage area is divided into multiple sectors by the directional antennas at the BS. Next, successive interference cancellation at the receivers is used to achieve interference cancellation, and the next MIC is applied where the decoded symbols are removed from the superimposed signals which reduce the effective interference on each D2D pair. It is shown that MIC provides considerable energy savings. In [54] the optimal cooperative design of energy efficiency and spectral efficiency in NOMA is investigated. The energy efficiency-spectral efficiency relationship of a single cell NOMA is shown to be linear with the slope of bandwidth per unit transmit power when total transmit power is constant. Next, a precoding technique which can be utilized to mitigate the intercell interference is also proposed. The technique improves both the energy and spectral efficiencies. Furthermore, the single-cell NOMA is extended to network NOMA, where a distributed multi-user zero-forcing (ZF) precoding scheme is applied to users with weak channel conditions. Further, an interference mitigation technique which can be utilized in a small cell network using cooperative communication and game theory approach is elaborated in [55].

\subsection{Sleep Wake Modes}

Optimizing the sleeping and waking modes of the devices will enhance the energy efficiency of wireless networks [57-60]. By controlling the active mode of the BS using optimal algorithms, energy enhancement is obtained for NOMA enabled network in [56]. Here, the level of low transmit power which facilitates constant received power and allows BSs to go into deep sleep modes is discussed. Further, the optimal percentage of deep sleep BSs which will maximize the energy efficiency while meeting QoS requirements is addressed in [56]. Besides, the waking or sleeping modes of UEs can be utilized to maintain the state of charge of the battery of the UE above a certain threshold. For example, this strategy is helpful in a cooperative NOMA scheme where certain UEs are used frequently as relays during the transmissions between the source and destination and their state of charge of the battery can completely discharge. In this section, the recent research works on the power domain based NOMA were discussed and in the proceeding section, the energy efficient NOMA schemes proposed under the code domain based NOMA are presented.

\section{Code Domain Based NOMA}

\subsection{Sparse Code Multiple Access}

In most of the above researches, NOMA was implemented in the power domain. However, there is room for energy efficiency enhancement using code domain NOMA as well. It is mentioned in [61], that out of the tested NOMA schemes, sparse code multiple access (SCMA) show lowest bit error rate and outage probability at high interference channels and utilizes low transmit power. Hence, SCMA falls under an energy efficient spectrum efficient algorithm that requires further research insight. In [62], an algorithm is proposed to enhance energy efficiency using SCMA in the uplink. It is shown that the SCMA scheme provides extra multiplexing ability while optimizing the energy consumption which results in an energy efficient approach for the NOMA uplink scenario. Hence, the SCMA scheme can support an extra number of users with the help of non-orthogonal transmission thereby enhancing the average energy efficiency of a single user. Here a low complexity decoding algorithm is utilized which makes the transceiver hardware implementation less complex. 


\subsection{Space Time Block Coding}

Another coding scheme namely, space time block coding (STBC) [63], is used in enhancing both spectral efficiency and energy efficiency of NOMA. In [56], authors present a cooperative NOMA scheme utilizing STBC. Both orthogonal and non-orthogonal are used simultaneously with STBC and it is shown that both spectral efficiency and energy efficiency are enhanced.

\subsection{Multi User Shared Access}

Furthermore, multi user shared access (MUSA) is a code domain technique that can be used to optimize energy efficiency, throughput, simultaneous connections of a NOMA based wireless network. In $[70,71]$, MUSA is used in an IoT scenario where the massive number of users are accommodated simultaneously using the same radio resource. A collection of short length code sequences are used since they enable simple and steady successive interference cancellation and manage high user loads.

\section{Practical Aspects of Deploying Green NOMA}

The available research contributions on practical implementations [91] of energy efficient NOMA are discussed in this section. In [92], the energy efficiency of a practical heterogeneous cloud radio access network (HCRAN), adopting NOMA, is discussed. Further, the optimal number of BSs that maximize the energy efficiency of the HCRAN is presented for both micro and macro type BSs which allocate power assuming a practical channel model. The results indicate that the proposed NOMA for the HCRAN outperforms the conventional OFDMA schemes in terms of providing higher energy efficiency of up to four times. Further, it is shown that with a low power supply at the cloud based central station (CCS), twice the number of micro BSs can be served to provide an improved energy efficiency of up to 1.6 times compared with the macro BSs and remote radio heads, that achieve the same energy efficiency with high-power CCS. Besides, in [93], authors concentrate on the design of green BS assignment incorporating optimal power allocation. Here, the impact of the proposed solution against the densities of small BSs and users is addressed. Further, a practical implementation of a hybrid NOMA network by combining the concepts of non-orthogonal multiple access (NOMA) and orthogonal frequency division multiplexing and a heuristic resource allocation algorithm is proposed which includes a low complexity user clustering mechanism utilizing search and allocation approach [94].

Another practical scenario of enhancing energy efficiency is discussed in [95], where SWIPT is utilized in a multiple input single output (MISO) cognitive radio (CR) and NOMA based network. Here, a large population of power limited battery-driven devices are supported by the network. In contrast to most of the existing works, which use an ideal linear energy harvesting model, in this study, a more practical non-linear energy harvesting model is adopted. Furthermore, aspects of secure communication are also added and the power efficiency of the network is maximized under practical secrecy rate and energy harvesting constraints. In order to improve the secrecy rate of secondary users [96], techniques of multiple antennas, cooperative relaying, jamming and artificial noise aided techniques are used. The transmission beamforming and artificial noise aided covariance were jointly optimized to satisfy secrecy rates of both primary and secondary users [96] and energy harvesting of receivers. Power optimization was carried under both perfect CSI and bounded CSI error model. The target of establishing secure communication and outperforming energy efficiency of OMA was accomplished with the proposed algorithm. In [97], authors address an IoT scenario where constant replacement of batteries is required for increasing the lifetime of IoT devices and maintain the network functionality. This challenge was practically solved using wireless power transfer mechanisms and here authors use wireless powered communication based mechanisms for the field-deployed IoT sensor network. Further, practical aspects of deployment of large scale NOMA network for massive Machine-Type Communication (mMTC) is addressed in [98]. Here, several practical challenges of large scale deployment of NOMA, namely the inter-NOMA-interference (INI), inter-cell interference 
and hardware implementation complexity is discussed. Next, diversity gain techniques are used to reduce the complexity of successive interference cancellation and compensate the severe degradation of coding gain.

\section{Trending Schemes for Energy Efficient NOMA}

In this section, various energy efficiency enhancing techniques are investigated and their applicability to the requirements of emerging wireless networks is discussed. Besides, the proposed and extended schemes of enhancing energy efficiency of NOMA based wireless networks are illustrated in Figure 5. Next, incorporating emerging trends such as modulation coding, constellation shaping, backscatter communication, channel coding, cloud, edge computing, caching, unmanned aerial vehicle (UAV), artificial intelligence (AI) and tactile internet (TI) to enhance energy efficiency in NOMA based wireless networks is detailed as follows.

\subsection{Asymmetric Modulation as a Precoding Technique for Self Interference Cancellation}

High interference level of NOMA is a resultant of enabling each user to access all the subcarrier channels. Precoding schemes designed to reduce the interference and complexity of signal decoding of the NOMA system can achieve a drastic energy efficiency than conventional NOMA [99-102]. Furthermore, research work on using asymmetric quadrature amplitude modulation (AQAM) based precoding schemes is limited. AQAM has several strong benefits that could be exploited to improve wireless communication systems which needs further attention $[103,104]$. The complexity of SIC in NOMA utilizing modulation based precoding is drastically reduced due to the addition of interference cancellation aspects to the constellation mapping prior to transmitting signals to the channel in $[46,99,105,106]$. Further, these precoding schemes will be beneficial in large scale dense mobile cellular networks. We propose AQAM as a feasible precoding technique for enhancing energy efficiency of NOMA. When using AQAM, similar to conventional NOMA, power gets allocated to users according to channel gains in an inversely proportional manner so that the best user gets the minimum power and the weakest user gets the maximum power. However, in contrast to the conventional NOMA, two changes are done to the constellation mapping at the BS for each user namely,

1. Incorporating the amount of transmitted power allocated to each user to the amplitude of the symbols in the constellation map such that the power level is proportional to amplitude. The UEs which are located far from the BS will get more power and hence the amplitude of the symbols which are equidistant from the origin will be higher. Hence, for the near UE, the amplitude will be lesser.

2. Mapping each user constellations asymmetrically that no two users have the same amplitude and phase. The constellation map of each user will be phase shifted by a predetermined angle which will incorporate a unique mapping to each user.

Thus, basically a precoding technique will be utilized here to insert self interference cancellation with the modulation scheme which will minimize the complex computing at the end user. This method is extremely useful in scenarios where the channel gains are equal or nearly equal. In the conventional NOMA scheme it is difficult to differentiate the users with equal strength channels. Therefore, the proposed built in self interference cancellation techniques can be a productive solution for the large scale mobile networks. Consider a system which used a QPSK modulation scheme initially, to transmit symbols under NOMA scheme for 3 users scenario. The new constellation diagram of each user under this proposed technique is illustrated in Figure 6. Here, the users are classified as farthest user, intermediate user and nearest user. The farthest user is allocated a constellation with the highest amplitude, $d_{1}$ and a constellation is rotated by an angle of $\theta$. Further, the intermediate user constellation is allocated a medium amplitude $d_{2}$ and a rotation by an angle of $\alpha$. The nearest user is allocated the minimum amplitude, $d_{3}$ and a rotation by $90^{\circ}$. 


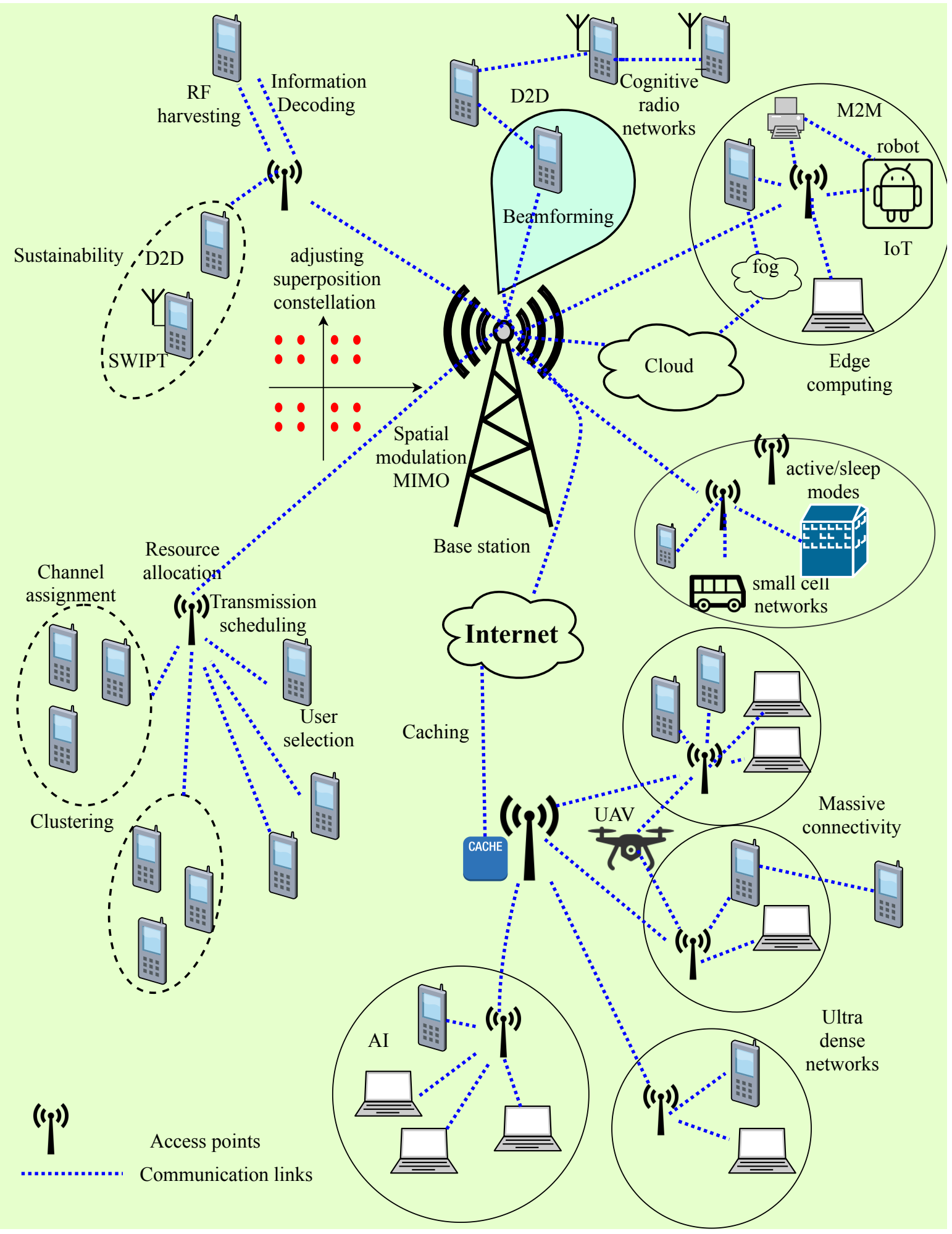

Figure 5. Proposed and extended schemes of enhancing energy efficiency of NOMA based wireless networks such as optimal resource allocation, sleeping modes, RF energy harvesting, cooperative communications (D2D, M2M), cloud computing, caching, beamforming, AI, UAV, modulation optimization. 


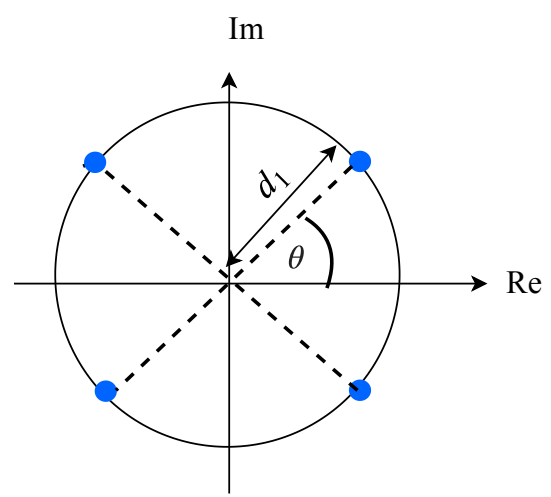

Farthest user (weak channel) Constellation map

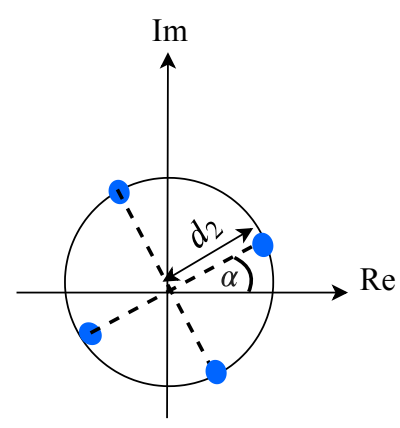

Intermediate user Constellation map

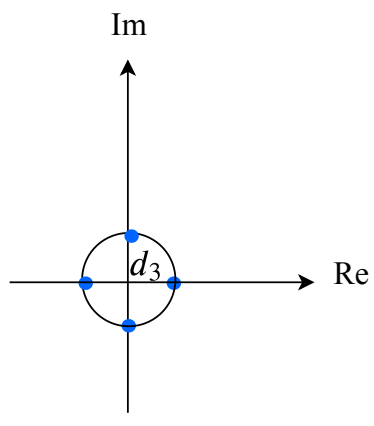

Nearest user (strong channel)

Constellation map

Figure 6. A sample constellation mapping design for 3 users under NOMA principle, where each user has a unique constellation mapping. A unique constellation map is assigned to each user at the transmitter in order to reduce the inter-symbolic interference and the complexity of decoding at the end receiver.

\subsection{Spatial Shift Keying Modulation}

Incorporating spatial modulation on NOMA is a feasible strategy of enhancing energy efficiency in massively connected wireless networks. The spatial modulation technique is a recently developed concept that is applied in multi antenna scenarios such as MIMO. It is beneficial in enhancing signal to noise ratio performance over the conventional modulation schemes. Hence, through recent research, it has been concluded that energy efficiency, spectral efficiency and capacity of a MIMO system can be enhanced using spatial modulation technique [107-109]. However, although several types of research have incorporated NOMA with spatial modulation [110-112], there is limited research available on the energy efficiency which can be additionally attained by mitigating inter-user interference present in spatial modulation. Further, since the computational complexity of the spatial modulation scheme is lower than other modulation schemes, the energy efficiency is higher than conventional MIMO NOMA [108,113]. In spatial modulation, the index of the transmitting or receiving antennas is used to denote extra information. The concept of spatial shift keying (SSK) was born using the spatial modulation concept $[111,114]$. In SSK, the transmission of information is done using the antenna index instead of using conventional amplitude or phase modulation techniques. While achieving almost same results as the conventional modulation techniques, this results in the removal of transceiver architecture elements such as coherent detectors [115]. Further, transmit beamforming is a promising technique for interference mitigation in spatial modulation. Also, it is important to allocate antennas to different users by considering the user requirements and channel conditions. With the spatial modulation scheme, the weak channels are less influenced by signal to noise ratio. Detecting the index of the antenna is comparatively less energy consuming which is beneficial for weak users. In addition, when considered with the conventional NOMA, the transceiver architecture and computation complexity have decreased enhancing the overall energy efficiency The Figure 7 illustrates incorporating spatial modulation and beamforming on a massive MIMO network. 


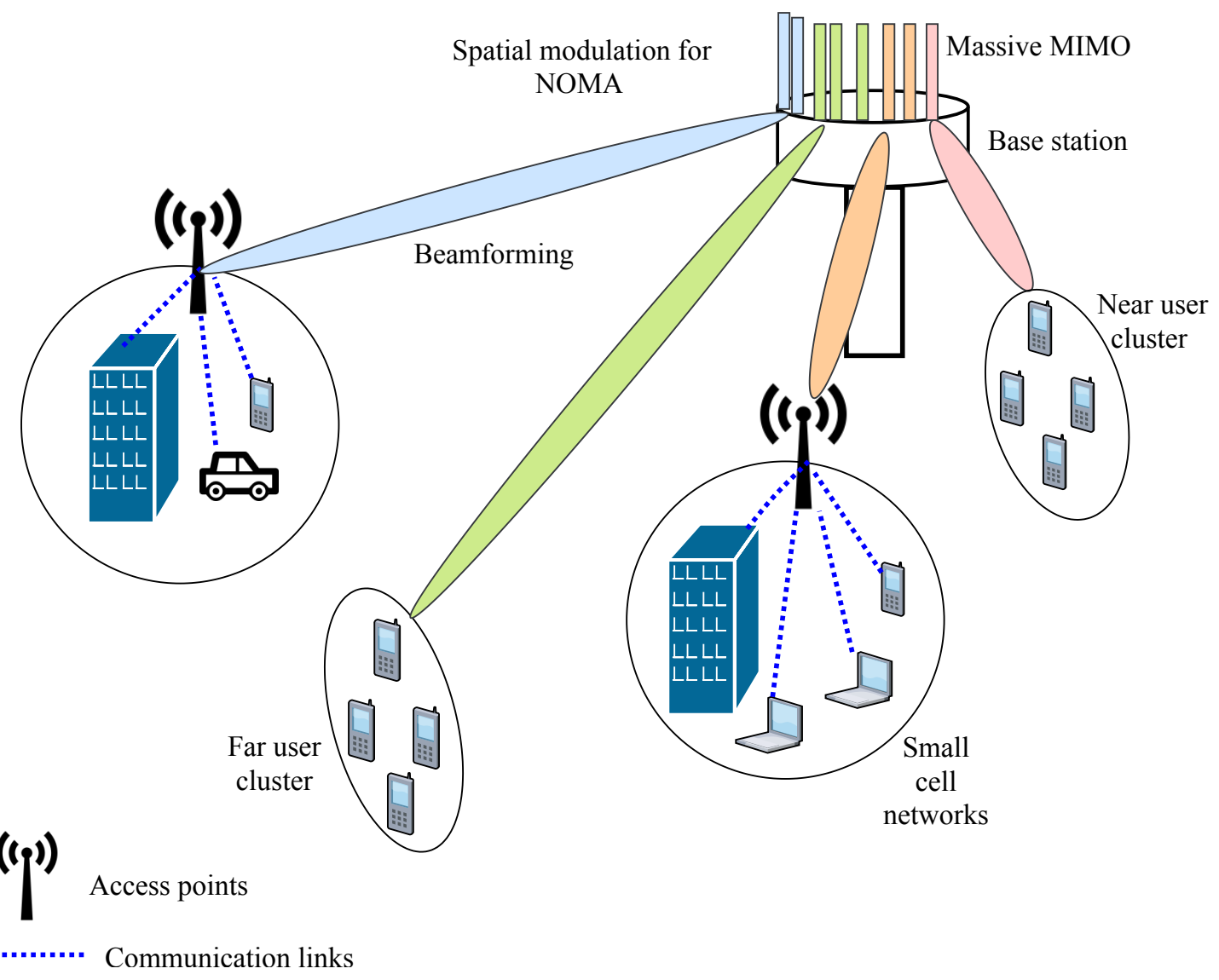

Figure 7. Spatial modulation scheme under NOMA principle, where the spatial diversity of the Massive MIMO BS is utilized to mitigate inter user interference and serve many users simultaneously.

\subsection{Constellation Shaping}

For a specific channel, there exists a signal distribution which maximizes the information rate [116]. Moreover, constellation shaping is a method of shaping the signal distribution so that both the information rate and energy efficiency is optimized. Hence, it is considered a more energy and spectral efficient way of reducing bit error rate and improving the capacity of the channel. It is used in digital modulation schemes such as amplitude and phase-shift keying (APSK) and the conventional quadrature amplitude modulation (QAM). There are two types of constellation shaping namely geometrical and probabilistic. The geometrical shaping scheme places points in the constellation mapping in non-uniform locations while making each symbol equally probable. However, the probabilistic constellation shaping scheme keeps the location of points of the constellation uniform but makes the probability of each symbol non-uniform which means that each symbol is sent with different probabilities [117]. This is employed in communication to limit the probability of occurrence of the high energy symbols and then increases the signal to noise ratio for all other symbols. Thus, low energy symbols will be transmitted more frequently than high energy symbols. Therefore, the low amplitude constellation points are sent with a higher probability than the high amplitude points. Additionally, the constellation points under the same amplitude layer are sent equally likely. Hence, the average symbol power will be decreased. However it will also reduce the average information transfer rate. Furthermore, mapping and demapping of the constellation points when using the constellation shaping method poses a significant challenge. As a solution, a bit sequence is mapped to a sequence of symbols whose probability of symbols per sequence is known correctly. In demapping, the principle of 'reverse concatenation' is used at the decoder to reduce complexity. However, the hardware implementation remains a considerable challenge [118]. Probability shaping has been used in the quadrature amplitude modulation (QAM) of a SWIPT scheme in [119]. It has shown that the energy 
efficiency of the SWIPT scheme based on constellation shaping performs better than the conventional SWIPT scheme based on power splitting mechanism.

\subsection{Ambient Backscatter Communication}

Ambient backscatter transmission is a recent communication mechanism that enables devices to communicate by harvesting energy from the nearby radio frequency signals and utilizes the energy to send information to nearby devices [120-122]. Since backscatter does not require battery power or or built in power infrastructure, it is an energy efficient communication method for emerging massive wireless networks. Specifically applications like D2D, IoT can be improved further with this scheme [123]. As there are limited research works conducted under energy efficient communications based on NOMA with backscatter, it is a feasible direction for future green wireless networking. One of the main challenges of backscatter communication, the reduced strength of the backscatter signal, can be overcome by full duplex communications as suggested in [124]. Moreover, research works reveal that frequency modulated (FM) signals also can be used for radio frequency energy harvesting [125]. Further, Wifi consuming low power can be generated using backscatter communications which is an energy efficient communication solution for the NOMA based massive connectivity applications [126]

\subsection{Channel Coding}

The channel coding mechanisms are used in communication systems to correct the errors caused due to noise, interference and poor signal strength of the communication channel [127]. Polar codes and LDPC have been proposed as the optimal error correction mechanisms for emerging 5G [64,67] and here we propose the research work which is useful in implementing energy efficiency under channel coding for NOMA based networks [65,66]. Energy efficient LDPC designs have been addressed in $[68,69]$ which can be utilized for NOMA networks to enhance energy performance.

\subsubsection{Low Density Parity Check (LDPC)}

LDPC codes with iterative belief propagation decoding promises to perform very close to Shannon's capacity limit. The nonzero elements distribution in the parity check matrix determines the performance of the LDPC code. Long LDPC codes are showing capacity approaching performance on many channels. Further, binary LDPC codes with iterative belief propagation decoding, do not perform well at short to moderate block lengths. Recently, protograph-based LDPC codes have shown to perform well under belief propagation decoding at short-to-moderate block length. Protograph-based LDPC has very low decoding complexity under iterative decoding algorithms. Non-binary LDPC codes also perform very close to the finite length performance limit where decoding complexity poses a major drawback. Also, some other branches of protograph codes, such as protograph-based raptor-like (PBRL) codes and spatially-coupled protograph-based (SCPB) codes, have been developed. Hence, protograph LDPC codes are considered as the new major direction for channel coding [127].

For the emerging $5 \mathrm{G}$ networks the usage of polar codes has been proposed as an optimal error correction mechanism because they have simple encoding decoding algorithms and have been proven to achieve channel capacity. Hence, NOMA based networks that are utilizing polar code sequences can utilize simple polar code decoders to enhance energy efficiency and increase the reliability of the information. Further, the research work conducted under channel coding is limited on how to enhance energy efficiency using polar coding in 5G especially in scenarios of massive machine-type communication (MTC) and ultra-reliable low-latency communication (URRLC) [66].

\subsubsection{Polar Coding}

Polar coding is a binary input discrete memoryless channel capacity achieving coding mechanism which uses a low complexity encoding and decoding algorithm as the code lengths go to infinity. Here, the channel polarization technique is used to construct the polar codes. The block code translates the available set of $\mathrm{N}$ independent and identical uses of binary input memoryless channel into two 
polarized types with capacities close to zero or one. The message is sent over the near-perfect channels and the useless channels are discarded. Under successive cancellation, for sufficiently large code word lengths, the block error probability decays exponentially. However, the recursive nature of the decoding and butterfly operation results in a large latency. This is improved using a technique called successive cancellation list (SCL) where all the decoding paths are listed. In addition, since there are several decoding path options there is no need to follow only one decoding path which improves the decoding efficiency. Improved versions of SCL include CRC- aided SCL, where the message is encoded with a cyclic redundancy check (CRC) and error detection.An improvement to SCL is CRC-aided SCL (CA-SCL), where the message is encoded with a CRC error detection code, and the result is polar coded [128]. Then at the decoding end, the concatenated CRC checksum is used to select the correct decoding path out of the SCL.

\subsection{Caching}

With the increased usage of the internet in the past two decades, the internet backhaul created massive data traffic in communications networks and the ultimate solution was web caching. Web caching is a technique where the content which is reused repeatedly by the end users is stored at intermediate nodes in the network rather than at the original remote server. This technique enabled to reduce retransmissions of data, enhance the performance metrics of the network and also provide a high quality of service (QoS) to the users [87,129]. Further, an illustration of a mobile cellular network with and without caching and its effect on data congestion in the network is shown in Figure 8.

Further, many research works reveal that caching enabled networking can enhance the system performance [129-138]. Web caching led to content delivery networks (CDNs) that are capable of replicating content across the internet and reducing more than $50 \%$ of the data traffic that is directed to the remote main servers which helped in reducing the amount of backhaul traffic in the network [129]. Further, enabling caching in wireless networks is another means of achieving energy efficiency in the emerging networks [87,129-135]. The power consumption for data transfer from a remote main server to the end user is higher than the data transfer from an intermediate cache node to the end user. However, here the energy consumption at the cache node for buffering the data for a long time is comparatively higher than an average node which is one of the drawbacks of caching. Therefore, caching is a promising energy efficient technique that can be incorporated with massive connectivity requirements. In literature, there remains limited research work on energy enhancing in NOMA using caching. Hence, NOMA related challenges such as massive content delivery with higher energy efficiency can be mitigated by techniques such as cache enabled intermediate relays in small cell networks, D2D transmitters, cache enabled edge and fog computing and artificial intelligence (AI) enabled caching. 


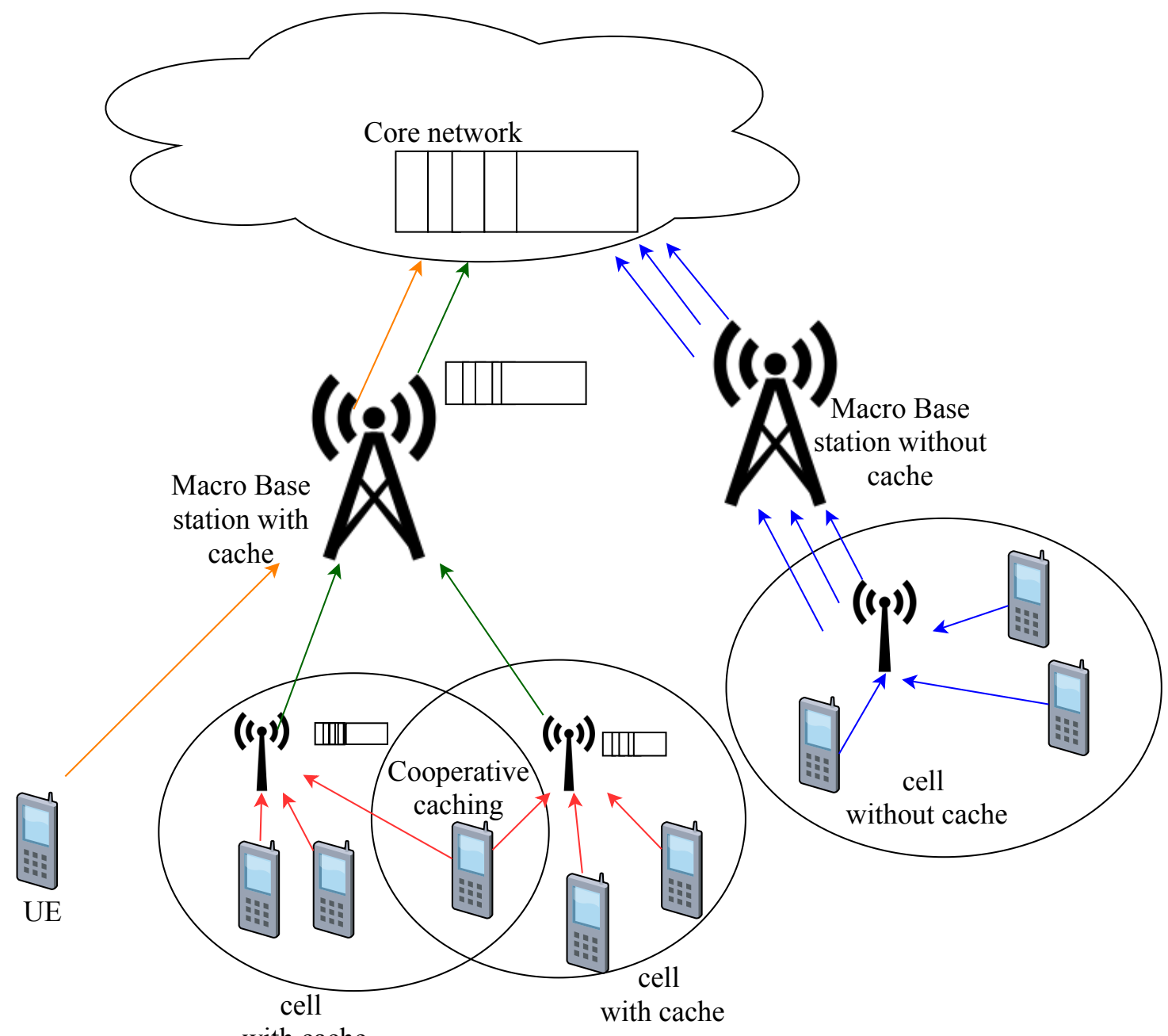

with cache

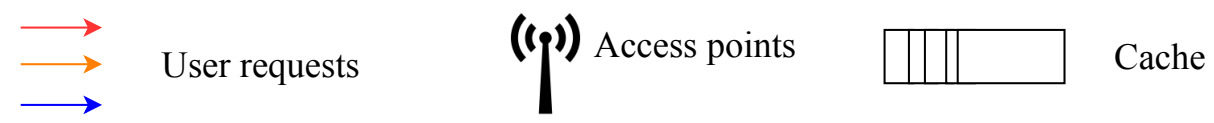

Figure 8. Effect of with and without caching on the data congestion in a mobile cellular wireless network.

\subsection{Computing}

To reduce the overall energy consumption for operations such as computations, communications and storage, types of computing provide effective solutions. Cloud, edge and fog computing plays a major role in networks where massive data communication is required. The overall view of all these three types of computing techniques and their connections in a wireless network is illustrated in Figure 9. 


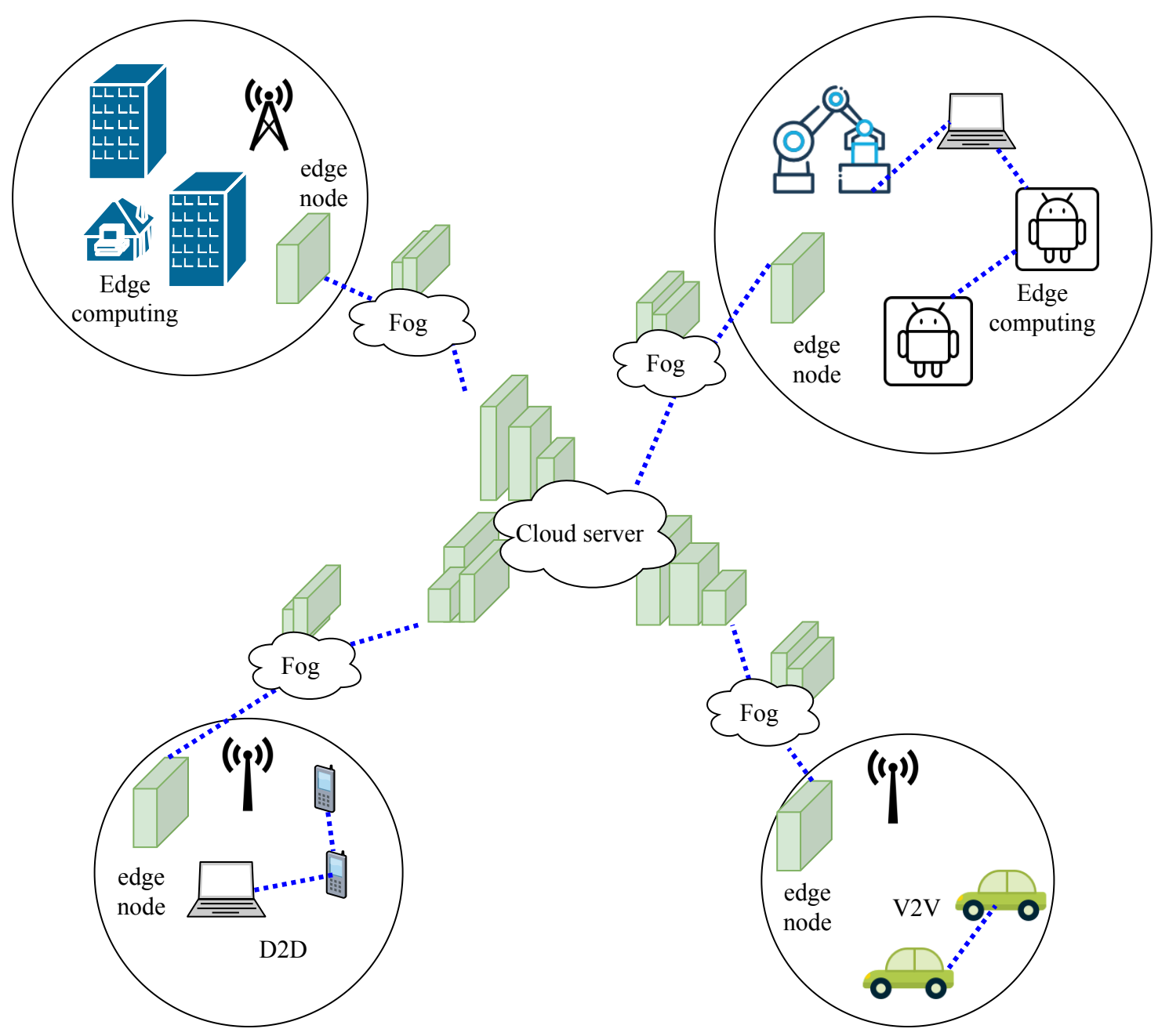

Figure 9. Overall view of three types of computing techniques namely, cloud, fog and edge and their connections and applications in a wireless network.

In this figure we elaborate on the recent trends under the computing categories and discuss achieving energy efficiency especially for NOMA based systems using computing. The difference between the computing types is the location where data processing occurs. Cloud computing processes data at a cloud server, while fog computing and edge computing perform the data processing near to the user. Cloud computing does not do real time data processing, security, and privacy. Further, in fog computing, data is processed at the land area network (LAN) node level or gateway. Fog computing bridges the connection between the cloud server and the network. In contrast, edge computing is processed on the embedded systems of the network and very close to the end device [139].

\subsubsection{Cloud Computing}

Cloud computing can deliver services to reduce on device computing, storage and communications by utilizing online data centers [140]. Cloud computing enabled energy efficient NOMA network is addressed in [141,142], where the cloud is used for baseband data processing and radio resource controlling to reduce the workload on the low power nodes, which are used to control data traffic in ultra-dense cellular networks. Furthermore, an energy efficient scheme which utilizes both cloud computing architecture and caching techniques is detailed in [143]. Cloud computing provides cooperative processing and network gains which helps in mitigating the operating expenses and the energy consumption of the wireless network infrastructure. 


\subsubsection{Fog computing}

Fog computing involves data processing in the middle layer between the cloud server and smart end devices at the edge. There is much research available on energy efficiency in fog computing in wireless networks [144-146]. Although there is considerable research on fog computing based energy efficiency for NOMA [147-150], it can be utilized further to mitigate the prevalent challenges such as resource optimization, low processing power and optimal placing of servers $[145,146]$. Fog computing is extremely useful in scenarios where low latency, high data rates, real time processing, reliability, storage are required. For example in scenarios such as industrial IoT and health, optimized fog computing designs are required $[145,151]$. Further, energy efficiency achieving caching schemes for fog computing is addressed in [152]. Therefore, NOMA based networks can utilize energy efficient fog computing designs to further improve the performance metrics.

\subsubsection{Edge Computing}

In edge computing, data is processed at a wireless network edge before being sent to a data center or a cloud. This reduces the stress of computation and traffic on the upper layer servers such as fog and cloud. Here the edge node, which is the automated controller at the network edge, is developed for computation. In terms of energy, edge computing nodes require lower processing power and since it reduces the computations at the upper layer servers, it will enhance the overall network energy consumption. Energy efficiency for NOMA using edge computing has been sought in [153,154]. Mobile edge computing incorporated with NOMA can serve multiple users using the same bandwidth and time [155]. Further, the computing done at the edge of the network results in a significant reduction in network traffic and resource utilization and an enhancement in energy efficiency. However, computing algorithms need to be further optimized for better efficiency.

\subsection{Unmanned Aaerial Vehicle (UAV)}

UAV communication has become a frontier topic for the upcoming mobile network due to its many applications and uses. Mostly in cellular wireless networks, UAV can be utilized as a BS or relay station which can improve the QoS of the network. There are several research contributions on utilization of UAV in cellular NOMA based networks [156,157]. In [157], authors address the challenge of full duplex communication in a UAV assisted NOMA network where cooperative UAV communication is utilized to enhance the performance of the network in terms of outage probability. Further, an energy efficiency enhancement for a NOMA enabled UAV network is addressed in [158]. Here, the authors utilize a UAV based aerial base station (UAV-BS) to provide a solution for the high data traffic demands of the future wireless networks. Since UAV is having limited energy reserves, efficient utilization of its energy is of significant importance. Here, the authors present an optimal scheme for the placement of UAV-BS systems such that energy efficiency of the UAV assisted scheme is enhanced. This proposed optimal scheme outperforms the conventional cooperative NOMA scheme with half duplex operation. Moreover, research work in [147], proposes an optimal subchannel assignment and power allocation for a fog computing based UAV assisted wireless network which can enhance energy efficiency of the system. Furthermore, energy efficiency ventures using UAV for a NOMA based networks are still at the beginning phase and can be further investigated.

\subsection{Artificial Intelligence (AI)}

AI schemes have become applicable for any field and have become an extremely useful technique in reducing high computational complexity. It is identified as a performance enhancement tool which can achieve intelligence in network operations, optimization, maintenance and management in the emerging mobile networks [159-161]. However, there are limited research work $[105,162]$ on improving the energy efficiency of NOMA based networks using branches of AI which leaves space to explore more solutions. In the paper [105], authors have addressed an optimization of energy 
efficiency and RF energy harvesting using a machine learning based algorithm. This algorithm is applied on a built in energy efficient modulation based system (BEEM-NOMA). The combination of BEEM-NOMA and machine learning outperforms the conventional NOMA in terms of energy efficiency. Moreover, in contrast to the conventional methods of optimizing a power allocation algorithm, a deep learning based approach to determine an approximated optimal solution is utilized in [162]. The results of the proposed scheme have shown to achieve a similar performance to the conventional optimization method. NOMA energy efficiency can further be improved by incorporating concepts such as distributed learning, reinforcement learning which can be used for massive networks. Possible ventures using AI include strong user symbol prediction, interference cancellation, cooperative energy harvesting, etc which can be used to enhance the energy efficiency of NOMA.

\subsection{Age of Information (AoI)}

An upcoming metric of evaluating the performance of wireless networks is AoI [163]. AoI in wireless networks is a measurement of information freshness. Thus, it is the main goal of the emerging network designs to incorporate AoI to improve the freshness of data received by the users. From the recent research work, it has been concluded that the AoI is higher in NOMA than OMA [164]. Hence, many research works have investigated optimizing AoI using different techniques [165] while there are fewer researches on enhancing energy efficiency of NOMA under AoI constraints [166]. AoI is higher in NOMA mainly due to the higher computational complexity which should be addressed properly to minimize AoI. Thus, various techniques discussed above related to AI, interference reduction, caching can be utilized to maintain the balance between AoI, energy and spectral efficiency of NOMA.

\subsection{Internet of Things (IoT)}

Application of NOMA in IoT can be elevated using fog computing, caching, edge computing, AI techniques. However, the trade-off between enhancing energy efficiency and data rates should be optimized. In the paper [123], a self-powered IoT network with NOMA is presented where the time allocation for wireless power and information transfer stages are optimized under node deployment and constraints for data transmission. M2M communication is a constituent of IoT networks and is becoming a more frequent technology. Due to the advance in cellular communications, cellular enabled $\mathrm{M} 2 \mathrm{M}$ is a convenient solution to M2M communication based systems. The challenges of serving multiple users, multiple transmissions at the same frequency and time, reducing data traffic and satisfying high energy efficiency requirements can be solved using NOMA scheme. In [167], authors propose an energy efficient NOMA scheme for M2M based IoT scenario. Here, UEs are configured as mMTC gateways. Next, game theory principles [168] are utilized to build an optimal power allocation scheme incorporating convex optimization principles.

\subsection{Tactile Internet(TI)}

TI is expected to enable low latency in the communication which will be useful in many applications related to health, industrial, business, entertainment sectors which require real time processing, automation to achieve the expected performance of the system $[169,170]$. Few research works have incorporated TI with NOMA [171,172]. However, enabling both TI with energy efficiency is a significant concern. In [173], enhancing energy efficiency while incorporating characteristics of TI is discussed. However, the application of TI specifically to NOMA systems incorporating energy efficiency remains low. In [174], authors discuss enhancing the performance of a NOMA based TI-IoT system. Furthermore, a feasible design of a energy efficient NOMA based network incorporating several emerging strategies is illustrated in the following Figure 10. 


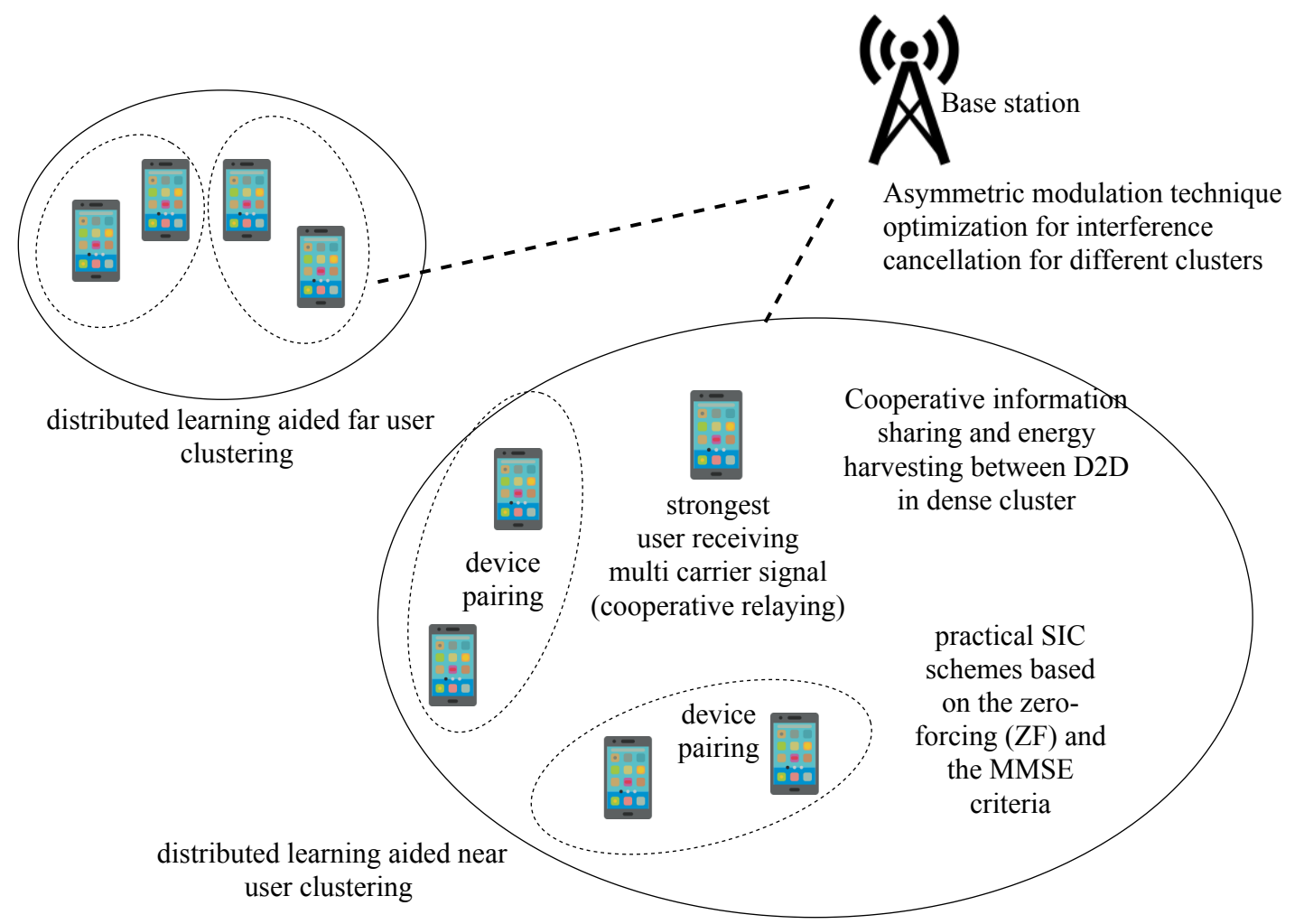

Figure 10. Incooperating several emerging energy efficient techniques together to achieve energy efficiency in NOMA based wireless networks.

\section{Conclusions}

Achieving system performances of high throughput, data rates, while optimizing energy efficiency will pose a huge challenge with the emerging large scale mobile wireless networks. It is expected that the cost of energy for the operation of the networks' multiple access schemes will increase drastically with the new technologies enabled with the emerging mobile networks. Therefore, the goal of this paper is to address the different methods of optimizing energy efficiency for NOMA based 5G green wireless communication networks available in literature. Although many methods are supporting energy efficient green communication for the 5G network, many challenges still exist which need attention. Hence, we proposed several trending strategies in this paper which will give an insight and future direction on enhancing the energy efficiency of NOMA.

Author Contributions: Conceptualization, V.B. and D.N.K.J.; original draft preparation, V.B.; supervision, D.N.K.J.; co-supervision, V.S., N.S., P.M. and H.M. All authors have read and agreed to the published version of the manuscript.

Funding: This work was funded, in part, by the Scheme for Promotion of Academic and Research Collaboration (SPARC), Ministry of Human Resource Development, India under the SPARC/2018-2019/P145/SL, in part, by the framework of Competitiveness Enhancement Program of the National Research Tomsk Polytechnic University, Russia.

Conflicts of Interest: The authors declare no conflict of interest

\section{Abbreviations}

The following abbreviations are used in this manuscript: 


\begin{tabular}{|c|c|}
\hline Abbreviation/Acronym & Definition \\
\hline AI & Artificial intelligence \\
\hline AoI & Age of Information \\
\hline BER & Bit error rate \\
\hline BEEM-NOMA & Built in energy efficient modulation based NOMA \\
\hline $\mathrm{BP}$ & Belief propagation \\
\hline BS & Base station \\
\hline CA-SCL & CRC-aided SCL \\
\hline CCS & Cloud based cloud station \\
\hline CoMP & Coordinated multi point networks \\
\hline CR & Cognitive radio \\
\hline CRC & Cyclic redundancy check \\
\hline CSI & Channel state information \\
\hline $\mathrm{D} 2 \mathrm{D}$ & Device to device \\
\hline HCRAN & Heterogeneous cloud radio access network \\
\hline IoT & Internet of Things \\
\hline INI & Inter-NOMA-interference \\
\hline IPMMSE & Interference predicted minimum mean square error \\
\hline LDPC & Low density parity check \\
\hline LTE & Long-Term Evolution \\
\hline MC-NOMA & Multi-carrier NOMA \\
\hline MIC & Multiple interference cancellation \\
\hline MIMO & Multiple input multiple output \\
\hline MISO & Multiple input single output \\
\hline M2M & Machine to machine \\
\hline M-NOMA & Modulation based NOMA \\
\hline MMSE & Minimum mean square error \\
\hline mMTC & Massive machine type communication \\
\hline NOMA & Non orthogonal multiple access \\
\hline OFDMA & Orthogonal frequency division multiple access \\
\hline OMA & Orthogonal multiple access \\
\hline PBRL & Protograph-based raptor-like \\
\hline PS & Power splitting \\
\hline QoS & Quality of services \\
\hline QPSK & Quadrature phase shift keying \\
\hline SC & Successive cancellation \\
\hline SCL & Successive cancellation list \\
\hline SCPB & Spatially-coupled protograph-based \\
\hline SCMA & Sparse code multiple access \\
\hline $\mathrm{SISO}$ & Single input single output \\
\hline SM & Spatial modulation \\
\hline SSK & Spatial shift keying \\
\hline SWIPT & Simultaneous wireless information and power transfer \\
\hline SWIPT-CNOMA & SWIPT assisted cooperative NOMA \\
\hline $\mathrm{TI}$ & Tactile internet \\
\hline TS & Time switching \\
\hline T-SIC & Triangular successive interference cancellation \\
\hline UAV & Unmanned aerial vehicle \\
\hline UAV-BS & UAV based aerial base station \\
\hline UE & User equipment \\
\hline URRLC & Ultra-reliable low-latency communication \\
\hline WPCCNs & Wireless powered cooperative communication networks \\
\hline $\mathrm{ZF}$ & Zero forcing \\
\hline
\end{tabular}




\section{References}

1. Liu, G.; Jiang, D. 5G: Vision and Requirements for Mobile Communication System towards Year 2020. Chin. J. Eng. 2016, 2016, 1-8. [CrossRef]

2. Chin, W.H.; Fan, Z.; Haines, R. Emerging Technologies and Research Challenges for 5G Wireless Networks. IEEE Wirel. Commun. 2014, 21, 106-112. [CrossRef]

3. 5G Wireless Technology / Qualcomm. https://www.qualcomm.com/invention/5g (accessed on 6 December 2019).

4. More than 50 Billion Connected Devices-Taking Connected Devices to Mass Market and Profitability. https://www.akos-rs.si/files/Telekomunikacije/Digitalna_agenda/Internetni_protokol_ Ipv6/More-than-50-billion-connected-devices.pdf (accessed on 6 December 2019).

5. Hao, W.; Chu, Z.; Zhou, F.; Yang, S.; Sun, G.; Wong, K.K. Green Communication for NOMA-Based CRAN. IEEE Internet Things J. 2018, 6, 666-678. [CrossRef]

6. Ding, Z.; Liu, Y.; Choi, J.; Sun, Q.; Elkashlan, M.; I, C.; Poor, H.V. Application of Non-Orthogonal Multiple Access in LTE and 5G Networks. IEEE Commun. Mag. 2017, 55, 185-191. [CrossRef]

7. Saito, Y.; Kishiyama, Y.; Benjebbour, A.; Nakamura, T.; Li, A.; Higuchi, K. Non-Orthogonal Multiple Access (NOMA) for Cellular Future Radio Access. In Proceedings of the IEEE 77th Vehicular Technology Conference (VTC Spring), Dresden, Germany, 2-5 June 2013; pp. 1-5. [CrossRef]

8. Ding, Z.; Lei, X.; Karagiannidis, G.K.; Schober, R.; Yuan, J.; Bhargava, V.K. A Survey on Non-Orthogonal Multiple Access for 5G Networks: Research Challenges and Future Trends. IEEE J. Sel. Areas Commun. 2017, 35, 2181-2195. [CrossRef]

9. Andrews, J.G.; Buzzi, S.; Choi, W.; Hanly, S.V.; Lozano, A.; Soong, A.C.K.; Zhang, J.C. What Will 5G Be? IEEE J. Sel. Areas Commun. 2014, 32, 1065-1082. [CrossRef]

10. Aldababsa, M.; Toka, M.; Gökceli, S.; Karabulut Kurt, G.; Kucur, O. A Tutorial on Nonorthogonal Multiple Access for 5G and Beyond. Wirel. Commun. Mob. Comput. 2018, 2018. [CrossRef]

11. Environmental Impact of Mobile Communications Networks/GSMA. https://www.gsma.com/ publicpolicy/wp-content/uploads/2012/04/environmobilenetworks.pdf (accessed on 10 December 2019).

12. Fehske, A.; Fettweis, G.; Malmodin, J.; Biczok, G. The global footprint of mobile communications: The ecological and economic perspective. IEEE Commun. Mag. 2011, 49, 55-62. [CrossRef]

13. Lubritto, C.; Petraglia, A.; Vetromile, C.; Curcuruto, S.; Logorelli, M.; Marsico, G.; D'Onofrio, A. Energy and environmental aspects of mobile communication systems. Energy 2011, 36. [CrossRef]

14. Zappone, A. A Survey of Energy-Efficient Techniques for 5G Networks and Challenges Ahead. IEEE J. Sel. Areas Commun. 2016, 34. [CrossRef]

15. Imran, M.; Khan, L.U.; Yaqoob, I.; Ahmed, E.; Qureshi, M.A.; Ahmed, A. Energy Harvesting in 5G Networks: Taxonomy, Requirements, Challenges, and Future Directions. arXiv 2019, arXiv:1910.00785. Available online: https: / / arxiv.org/abs/1910.00785 (accessed on 6 December 2019)

16. Fang, F.; Zhang, H.; Cheng, J.; Leung, V. Energy-efficient resource scheduling for NOMA systems with imperfect channel state information. In Proceedings of the IEEE International Conference on Communications (ICC), Paris, France, 21-25 May 2017. [CrossRef]

17. Zhang, Y.; Wang, H.M.; Zheng, T.X.; Yang, Q. Energy-Efficient Transmission Design in Non-Orthogonal Multiple Access. IEEE Trans. Veh. Technol. 2017, 66, 2852-2857. [CrossRef]

18. Fang, F.; Zhang, H.; Cheng, J.; Leung, V.C.M. Energy efficiency of resource scheduling for non-orthogonal multiple access (NOMA) wireless network. In Proceedings of the 2016 IEEE International Conference on Communications (ICC), Kuala Lumpur, Malaysia, 23-27 May 2016.

19. Zhu, J.; Wang, J.; Huang, Y.; He, S.; You, X.; Yang, L. On Optimal Power Allocation for Downlink Non-Orthogonal Multiple Access Systems. IEEE J. Sel. Areas Commun. 2017, 35, 2744-2757. [CrossRef]

20. Fang, F.; Zhang, H.; Cheng, J.; Leung, V.C.M. Energy-Efficient Resource Allocation for Downlink Non-Orthogonal Multiple Access Network. IEEE Trans. Commun. 2016, 64, 3722-3732. [CrossRef]

21. Lei, L.; Yuan, D.; Värbrand, P. On Power Minimization for Non-orthogonal Multiple Access (NOMA). IEEE Commun. Lett. 2016, 20, 2458-2461. [CrossRef]

22. Choi, J. Joint Rate and Power Allocation for NOMA with Statistical CSI. IEEE Trans. Commun. 2017, 65, 4519-4528. [CrossRef] 
23. Senel, K.; Tekinay, S. Optimal Power Allocation in NOMA Systems with Imperfect Channel Estimation. In Proceedings of the GLOBECOM 2017-2017 IEEE Global Communications Conference, Singapore, 4-8 December 2017. [CrossRef]

24. Zamani, M.; Eslami, M.; Khorramizade, M.; Ding, Z. Energy Efficient Power Allocation for NOMA with Imperfect CSI. IEEE Trans. Veh. Technol. 2018, 68, 1009-1013. [CrossRef]

25. Sun, Q.; Han, S.; I, C.; Pan, Z. Energy efficiency optimization for fading MIMO non-orthogonal multiple access systems. In Proceedings of the IEEE International Conference on Communications (ICC), London, UK, 8-12 June 2015; pp. 2668-2673. [CrossRef]

26. Zhang, H.; Wang, B.; Jiang, C.; Long, K.; Nallanathan, A.; Leung, V.C.M.; Poor, H.V. Energy Efficient Dynamic Resource Optimization in NOMA System. IEEE Trans. Wirel. Commun. 2018, 17, 5671-5683. [CrossRef]

27. Wei, Z.; Ng, D.W.K.; Yuan, J. Power-Efficient Resource Allocation for MC-NOMA with Statistical Channel State Information. In proceedings of the IEEE Global Communications Conference (GLOBECOM), Washington, DC, USA, 4-8 December 2016.

28. Ruby, R.; Zhong, S.; Ng, D.W.K.; Wu, K.; Leung, V.C.M. Enhanced Energy-Efficient Downlink Resource Allocation in Green Non-Orthogonal Multiple Access Systems. Comput. Commun. 2019, 139, 78-90. [CrossRef]

29. Uddin, M.F. Energy efficiency maximization by joint transmission scheduling and resource allocation in downlink NOMA cellular networks. Comput. Netw. 2019, 159, 37-50. [CrossRef]

30. Yang, Z.; Xu, W.; Xu, H.; Shi, J.; Chen, M. Energy Efficient Non-Orthogonal Multiple Access for Machine-to-Machine Communications. IEEE Commun. Lett. 2017, 21, 817-820. [CrossRef]

31. Wang, R.; Liu, G.; Zhang, H.; Kang, W.; Tsiftsis, T.; Leung, V.C.M. Resource Allocation for Energy-Efficient NOMA Network Based on Super-Modular Game. In proceedings of the IEEE International Conference on Communications Workshops (ICC Workshops), Kansas City, MO, USA, 20-24 May 2018; pp. 2474-9133. [CrossRef]

32. Zhang, H.; Fang, F.; Cheng, J.; Long, K.; Wang, W.; Leung, V.C.M. Energy-Efficient Resource Allocation in NOMA Heterogeneous Networks. IEEE Wirel. Commun. 2018, 25, 48-53. [CrossRef]

33. Zhai, D.; Zhang, R.; Cai, L.; Li, B.; Jiang, Y. Energy-Efficient User Scheduling and Power Allocation for NOMA-Based Wireless Networks With Massive IoT Devices. IEEE Internet Things J. 2018, 5, 1857-1868. [CrossRef]

34. Baidas, M.; Bahbahani, Z.; Alsusa, E. User-Association and Channel Assignment in Downlink Multi-Cell NOMA Networks: A Matching-Theoretic Approach. EURASIP J. Wirel. Commun. Netw. 2019. [CrossRef]

35. Singh, R. Sub-channel assignment and resource scheduling for non-orthogonal multiple access (NOMA) in downlink coordinated multi-point systems. In proceedings of the 20th Conference on Innovations in Clouds, Internet and Networks (ICIN), Paris, France, 7-9 March 2017. [CrossRef]

36. Ni, Z.; Chen, Z.; Zhang, Q.; Zhou, C. Analysis of RF Energy Harvesting in Uplink-NOMA IoT-based Network. In proceedings of the IEEE 90th Vehicular Technology Conference (VTC2019-Fall), Honolulu, HI, USA, 22-25 September 2019. [CrossRef]

37. Guo, W.; Wang, Y. Cooperative Non-Orthogonal Multiple Access with Energy Harvesting. Information 2017, 8, 111. [CrossRef]

38. Ding, Z.; Perlaza, S.M.; Esnaola, I.; Poor, H.V. Power Allocation Strategies in Energy Harvesting Wireless Cooperative Networks. IEEE Trans. Wirel. Commun. 2014, 13, 846-860. [CrossRef]

39. Chen, H.H.; Li, Y.; Jiang, Y.; Ma, Y.; Vucetic, B. Distributed Power Splitting for SWIPT in Relay Interference Channels using Game Theory. IEEE Trans. Wirel. Commun. 2014, 14. [CrossRef]

40. Yang, Z.; Pan, Y.; Xu, W.; Guan, R.; Wang, Y.; Chen, M. Energy efficient resource allocation for machine-to-machine communications with NOMA and energy harvesting. In Proceedings of the IEEE Conference on Computer Communications Workshops (INFOCOM WKSHPS), Atlanta, GA, USA, 23 November 2017.

41. Ha, D.B.; Agrawal, J. Performance Analysis for NOMA Relaying System in Next-Generation Networks with RF Energy Harvesting; IntechOpen: London, UK, 2019. [CrossRef]

42. Liu, Y.; Ding, Z.; Elkashlan, M.; Poor, H.V. Cooperative Non-orthogonal Multiple Access With Simultaneous Wireless Information and Power Transfer. IEEE J. Sel. Areas Commun. 2016, 34, 938-953. [CrossRef] 
43. Ding, Z.; Krikidis, I.; Sharif, B.; Poor, H.V. Wireless Information and Power Transfer in Cooperative Networks With Spatially Random Relays. IEEE Trans. Wirel.Commun. 2014, 13, 4440-4453. [CrossRef]

44. Ulukus, S.; Yener, A.; Erkip, E.; Simeone, O.; Zorzi, M.; Grover, P.; Huang, K. Energy Harvesting Wireless Communications: A Review of Recent Advances. IEEE J. Sel. Areas Commun. 2015, 33, 360-381. [CrossRef]

45. Yuan, Y.; Xu, Y.; Yang, Z.; Xu, P.; Ding, Z. Energy Efficiency Optimization in Full-Duplex User-Aided Cooperative SWIPT NOMA Systems. IEEE Trans. Commun. 2019, 67, 5753-5767. [CrossRef]

46. Rajaram, A.; Khan, R.; Tharranetharan, S.; Jayakody, D.N.K.; Dinis, R.; Panic, S. Novel SWIPT Schemes for 5G Wireless Networks. Sensors 2019, 19, 1169. [CrossRef] [PubMed]

47. Medepally, B.; Mehta, N.B. Voluntary Energy Harvesting Relays and Selection in Cooperative Wireless Networks. IEEE Trans. Wirel. Commun. 2010, 9, 3543-3553. [CrossRef]

48. Ye, Y.; Li, Y.; Wang, D.; Lu, G. Power splitting protocol design for the cooperative NOMA with SWIPT. In Proceedings of the 2017 IEEE International Conference on Communications (ICC), Paris, France, 31 July 2017. [CrossRef]

49. Tang, J.; Luo, J.; Liu, M.; So, D.; Alsusa, E.; Chen, G.; Wong, K.K.; Chambers, J. Energy Efficiency Optimization for NOMA with SWIPT. IEEE J. Sel. Top. Signal Process. 2019, 13, 452-466. [CrossRef]

50. Mehmood, K.; Niaz, M.T.; Kim, H.S. Dynamic Fractional Frequency Reuse Diversity Design for Inter-Cell Interference Mitigation in Non-Orthogonal Multiple Access (NOMA) Multicellular Networks. Wirel. Commun. Mob. Comput. 2018. [CrossRef]

51. Haci, H.; Zhu, H.; Wang, J. Performance of Non-orthogonal Multiple Access With a Novel Asynchronous Interference Cancellation Technique. IEEE Trans. Commun. 2017, 65, 1319-1335. [CrossRef]

52. Su, X.; Yu, H.; Kim, W.; Choi, C.; Choi, D. Interference cancellation for non-orthogonal multiple access used in future wireless mobile networks. EURASIP J. Wirel. Commun. Netw. 2016, 231. [CrossRef]

53. Gandotra, P.; Jain, S. Green NOMA with Multiple Interference Cancellation (MIC) using Sector Based Resource Allocation. IEEE Trans. Netw. Serv. Manag. 2018, 15, 1006-1017. [CrossRef]

54. E-Letter-September2014.pdf. Available online: http://mmc.committees.comsoc.org/files/2016/04/E-LetterSeptember2014.pdf (accessed on 22 December 2019).

55. Wang, X.; Zhang, H.; Tian, Y.; Ding, Z.; Leung, V.C.M. Locally Cooperative Interference Mitigation for Small Cell Networks with Non-Orthogonal Multiple Access: A Potential Game Approach. In Proceedings of the IEEE International Conference on Communications (ICC), Kansas City, MO, USA, 20-24 May 2018. [CrossRef]

56. Jamal, M.N.; Hassan, S.A.; Jayakody, D.N.K.; Rodrigues, J.J.P.C. Efficient Nonorthogonal Multiple Access: Cooperative Use of Distributed Space-Time Block Coding. IEEE Veh.Technol. Mag. 2018, 13, 70-77. [CrossRef]

57. El-Amine, A.; Iturralde, M.; Haj Hassan, H.A.; Nuaymi, L. A Distributed Q-Learning Approach for Adaptive Sleep Modes in 5G Networks. In Proceedings of the IEEE Wireless Communications and Networking Conference (WCNC), Marrakesh, Morocco, 15-18 April 2019 . [CrossRef]

58. Odadzic, B.; Dobrilovic, D.; Stojanov, Z.; Odadžić, D. The Cross Layer Model for Wireless Networks Energy Efficiency. Wirl. Commun. Mob. Comput. 2008, 9, 529-542, doi: 10.1002/wcm.698. [CrossRef]

59. Kang, M.W.; Chung, Y.W. An Efficient Energy Saving Scheme for Base Stations in 5G Networks with Separated Data and Control Planes Using Particle Swarm Optimization. Energies 2017, 10, 1417. [CrossRef]

60. Feng, M.; Mao, S.; Jiang, T. Base Station ON-OFF Switching in 5G Wireless Networks: Approaches and Challenges. IEEE Wirel. Commun. 2017, 24, 46-54. [CrossRef]

61. Elsaraf, Z.; Khan, F.; Ahmed, Q. Performance Analysis of Code-Domain NOMA in 5G Communication Systems. In Proceedings of the 2018 ELEKTRO, Mikulov, Czech Republic, 21-23 May 2018. [CrossRef]

62. Zhang, S.; Xu, X.; Lu, L.; Wu, Y.; Gaoning, H.; Chen, Y. Sparse code multiple access: An energy efficient uplink approach for 5G wireless systems. In Proceedings of the 2014 IEEE Global Communications Conference, Austin, TX, USA, 8-12 December 2014; pp. 4782-4787. [CrossRef]

63. Tarokh, V.; Jafarkhani, H.; Calderbank, A.R. Space-time block coding for wireless communications: performance results. IEEE J. Sel. Areas Commun. 1999, 17, 451-460. [CrossRef]

64. Sharma, A.; Salim, M. Polar Code Appropriateness for Ultra-Reliable and Low-Latency Use Cases of 5G Systems. Int. J. Netw. Distrib. Comput. 2019. [CrossRef]

65. Li, L.; Wang, Q.; Hu, Y.; Zhang, C. Energy Consumption of Polar Codes for Wireless Sensor Networks. In International Wireless Internet Conference 2017; Springer: Cham, Switzerland, 2017; pp. 140-149. [CrossRef] 
66. Ercan, F.; Tonnellier, T.; Gross, W.J. Energy-Efficient Hardware Architectures for Fast Polar Decoders. IEEE Trans. Circuits Syst. I Regul. Pap. 2019. [CrossRef]

67. Richardson, T.; Kudekar, S. Design of Low-Density Parity Check Codes for 5G New Radio. IEEE Commun. Mag. 2018, 56, 28-34. [CrossRef]

68. Nguyen, T.T.B.; Nguyen Tan, T.; Lee, H. Efficient QC-LDPC Encoder for 5G New Radio. Electronics 2019, 8, 668. [CrossRef]

69. Maunder, R. A Vision for 5G Channel Coding; AccelerComm White Paper; Accelercomm Ltd.: Southampton, UK, 2016.

70. Yuan, Z.; Yu, G.; Li, W.; Yuan, Y.; Wang, X.; Xu, J. Multi-User Shared Access for Internet of Things. In Proceedings of the 2016 IEEE 83rd Vehicular Technology Conference (VTC Spring), Nanjing, China, 15-18 May 2016. [CrossRef]

71. Yeom, J.S.; Chu, E.; Jung, B.C.; Jin, H. Performance Analysis of Diversity-Controlled Multi-User Superposition Transmission for 5G Wireless Networks. Sensors 2018, 18, 536. [CrossRef]

72. Lu, X.; Wang, P.; Niyato, D.; Kim, D.I.; Han, Z. Wireless Networks With RF Energy Harvesting: A Contemporary Survey. IEEE Commun. Surv. Tutor. 2015, 17, 757-789. [CrossRef]

73. Perera, T.; Jayakody, D.N.; Sharma, S.K.; Chatzinotas, S.; Li, J. Simultaneous Wireless Information and Power Transfer (SWIPT): Recent Advances and Future Challenges. IEEE Commun. Surv. Tutor. 2018, 20, $264-302$. [CrossRef]

74. Huang, K.; Zhou, X. Cutting the last wires for mobile communications by microwave power transfer. IEEE Commun. Mag. 2015, 53, 86-93. [CrossRef]

75. Tang, X.; Wang, X.; Cattley, R.; Gu, F.; Ball, A.D. Energy Harvesting Technologies for Achieving Self-Powered Wireless Sensor Networks in Machine Condition Monitoring: A Review. Sensors 2018, 18, 4113. [CrossRef] [PubMed]

76. Nintanavongsa, P.; Naderi, M.Y.; Chowdhury, K.R. Medium access control protocol design for sensors powered by wireless energy transfer. In Proceedings of the 2013 IEEE INFOCOM, Turin, Italy, 14-19 April 2013; pp. 150-154. [CrossRef]

77. Luo, Y.; Pu, L.; Wang, G.; Zhao, Y. RF Energy Harvesting Wireless Communications: RF Environment, Device Hardware and Practical Issues. Sensors 2019, 19, 3010. [CrossRef] [PubMed]

78. Pehlivan, I.; Coleri Ergen, S. Scheduling of Energy Harvesting for MIMO Wireless Powered Communication Networks. IEEE Commun. Lett. 2018, 23, 152-155. [CrossRef]

79. Alsharif, M.; Kim, S.; Kuruoğlu, N. Energy Harvesting Techniques for Wireless Sensor Networks/Radio-Frequency Identification: A Review. Symmetry 2019, 11, 865. [CrossRef]

80. Cui, S.; Goldsmith, A.J.; Bahai, A. Energy-efficiency of MIMO and cooperative MIMO techniques in sensor networks. IEEE J. Sel. Areas Commun. 2004, 22, 1089-1098. [CrossRef]

81. Jayaweera, S.K. Virtual MIMO-based cooperative communication for energy-constrained wireless sensor networks. IEEE Trans. Wirel. Commun. 2006, 5, 984-989. [CrossRef]

82. Wan, Z.G.; Tan, Y.K.; Yuen, C. Review on energy harvesting and energy management for sustainable wireless sensor networks. In Proceedings of the IEEE 13th International Conference on Communication Technology, Jinan, China, 25-28 September 2011; pp. 362-367. [CrossRef]

83. Sudevalayam, S.; Kulkarni, P. Energy Harvesting Sensor Nodes: Survey and Implications. IEEE Commun. Surv. Tutor. 2011, 13, 443-461. [CrossRef]

84. Lei, L.; Chang, Z.; Hu, Y.; Yuan, Y.; Chatzinotas, S. Energy-Efficient Resource Optimization with Wireless Power Transfer for Secure NOMA Systems. In Proceedings of the 2018 IEEE/CIC International Conference on Communications in China (ICCC), Beijing, China, 16-18 August 2018; pp. 106-110. [CrossRef]

85. Wang, S.; Shi, W.; Wang, C. Energy-Efficient Resource Management in OFDM-Based Cognitive Radio Networks Under Channel Uncertainty. IEEE Trans. Commun. 2015, 63, 3092-3102. [CrossRef]

86. Ali, Z.; Sidhu, G.A.S.; Waqas, M.; Gao, F.; Jin, S. Achieving energy fairness in multiuser uplink CR transmission. In Proceedings of the 2016 IEEE Wireless Communications and Networking Conference, Doha, Qatar, 3-6 April 2016; pp. 1-6. [CrossRef]

87. Usama, M.; Erol-Kantarci, M. A Survey on Recent Trends and Open Issues in Energy Efficiency of 5G. Sensors 2019, 19, 3126. [CrossRef] 
88. Klapez, M.; Grazia, C.A.; Casoni, M. Energy Savings of Sleep Modes Enabled by 5G Software-Defined Heterogeneous Networks. In Proceedings of the 2018 IEEE 4th International Forum on Research and Technology for Society and Industry (RTSI), Palermo, Italy, 10-13 September 2018. [CrossRef]

89. Fernández-Fernández, A.; Cervelló-Pastor, C.; Ochoa-Aday, L. Energy Efficiency and Network Performance: A Reality Check in SDN-Based 5G Systems. Energies 2017, 10, 2132. [CrossRef]

90. Varshney, L.R. Transporting information and energy simultaneously. In Proceedings of the IEEE International Symposium on Information Theory, Toronto, ON, Canada, 6-11 July 2008; pp. 1612-1616. [CrossRef]

91. Benjebbour, A.; Saito, Y.; Kishiyama, Y.; Li, A.; Harada, A.; Nakamura, T. Concept and practical considerations of non-orthogonal multiple access (NOMA) for future radio access. In Proceedings of the International Symposium on Intelligent Signal Processing and Communication Systems, Naha, Japan, 12-15 November 2013. [CrossRef]

92. Vien, Q.T.; Le, T.; Barn, B.; Van Ca, P. Optimising energy efficiency of non-orthogonal multiple access for wireless backhaul in heterogeneous cloud radio access network. IET Commun. 2016, 10, 2516-2524. [CrossRef]

93. Zhou, T.; Zhao, J.; Qin, D.; Li, X.; Li, C.; Luxi, Y. Green Base Station Assignment for NOMA-Enabled HCNs. IEEE Access 2019. [CrossRef]

94. Shi, J.; Yu, W.; Ni, Q.; Liang, W.; Li, Z.; Xiao, P. Energy Efficient Resource Allocation in Hybrid Non-Orthogonal Multiple Access Systems. IEEE Trans. Commun. 2019, 67, 3496-3511. [CrossRef]

95. Zhou, F.; Chu, Z.; Sun, H.; Hu, R.; Hanzo, L. Artificial noise aided secure cognitive beamforming for cooperative MISO-NOMA using SWIPT. IEEE J. Sel. Areas Commun. 2017, 36, 918-931. [CrossRef]

96. Shirvani moghaddam, S. Primary and Secondary Users in Cognitive Radio-Based Wireless Communication Systems; IntechOpen: London, UK, 2018. [CrossRef]

97. Li, X.; Luo, C.; Ji, H.; Zhuang, Y.; Zhang, H.; Leung, V. Energy consumption optimization for self-powered IoT networks with non-orthogonal multiple access. Int. J. Commun. Syst. 2019, 33, e4174. [CrossRef]

98. Hossain, E.; Al-Eryani, Y. Large-Scale NOMA: Promises for Massive Machine-Type Communication. arXiv 2019, arXiv:1910.00785.

99. Khan, R.; Jayakody, D.N.; Chen, B. Non-orthogonal multiple access: Basic interference management technique. Int. J. Eng. Technol. 2018, 7, 357-361. [CrossRef]

100. Wang, B.; Dai, L.; Wang, Z.; Ge, N.; Zhou, S. Spectrum and Energy Efficient Beamspace MIMO-NOMA for Millimeter-Wave Communications Using Lens Antenna Array. IEEE J. Sel. Areas Commun. 2017, 35, 2370-2382. [CrossRef]

101. Wei, Z. Performance Analysis and Design of Non-orthogonal Multiple Access for Wireless Communications. arXiv 2019, arXiv:1910.00946.

102. Sun, H. Spectral, Energy and Computation Efficiency in Future 5G Wireless Networks. Ph.D. Thesis, Utah State University, Logan, UT, USA, 2019.

103. Morelos-Zaragoza, R.; Kreb, E. Asymmetric Modulation for Cognitive Radio and Intelligent Environments. In Proceedings of the 2004 Software Defined RadioTechnical Conference and Product Exposition, Phoenix, AZ, USA, 17 November 2004.

104. Divsalar, D.; Simon, M.; Yuen, J. Trellis Coding with Asymmetric Modulations. IEEE Trans. Commun. 1987, 35, 130-141. [CrossRef]

105. Khan, R.; Dushantha, N.; Jayakody, D.N.; Sharma, V.; Kumar, V.; Kaur, K.; Chang, Z. A Machine Learning Based Energy-Efficient Non-Orthogonal Multiple Access Scheme. In Proceedings of the International Forum on Strategic Technology, Tomsk, Russia, 14-17 October 2019.

106. Khan, R.; Jayakody, D.N.; Pervaiz, H.; Tafazolli, R. Modulation Based Non-Orthogonal Multiple Access for 5G Resilient Networks. In Proceedings of the IEEE GlobecomWorkshops (GC Wkshps), Abu Dhabi, UAE, 9-13 December 2018. [CrossRef]

107. Ramarakula, M. Energy Efficiency and Capacity Analysis for Spatial Modulation in MIMO Systems. Int. J. Emerg. Technol. Innov. Res. 2018, 5, 985-992.

108. Mesleh, R.; Haas, H.; Sinanovic, S.; Ahn, C.W.; Yun, S. Spatial Modulation. IEEE Trans. Veh. Technol. 2008, 57, 2228-2241. [CrossRef]

109. Cogen, F.; Aydin, E. Hexagonal Quadrature Amplitude Modulation Aided Spatial Modulation. In Proceedings of the 11th International Conference on Electrical and Electronics Engineering, Bursa, Turkey, 28-30 November 2019. 
110. Siregar, R.F.; Murti, F.W.; Shin, S.Y. Combination of spatial modulation and non-orthogonal multiple access using hybrid detection scheme. In Proceedings of the 2017 Ninth International Conference on Ubiquitous and Future Networks (ICUFN), Milan, Italy, 4-7 July 2017; pp. 476-481. [CrossRef]

111. Zhu, X.; Wang, Z.; Cao, J. NOMA-Based Spatial Modulation. IEEE Access 2017, 5, 3790-3800. [CrossRef]

112. Li, Q.; Wen, M.; Basar, E.; Poor, H.V.; Chen, F. Spatial Modulation-Aided Cooperative NOMA: Performance Analysis and Comparative Study. IEEE J. Sel. Top. Signal Process. 2019, 13, 715-728. [CrossRef]

113. Zhong, C.; Hu, X.; Chen, X.; Ng, D.W.K.; Zhang, Z. Spatial Modulation Assisted Multi-Antenna Non-Orthogonal Multiple Access. IEEE Wirel. Commun. 2018, 25, 61-67. [CrossRef]

114. Humadi, K.; Sulyman, A.; Alsanie, A. Spatial Modulation Concept for Massive Multiuser MIMO Systems. Int. J. Antennas Propag. 2014, 2014, 1-9. [CrossRef]

115. Jeganathan, J.; Ghrayeb, A.; Szczecinski, L.; Ceron, A. Space shift keying modulation for MIMO channels. IEEE Trans. Wirel. Commun. 2009, 8, 3692-3703. [CrossRef]

116. Jia, Z.; Campos, L.A. Coherent Optics for Access Networks; CRC Press: Boca Raton, FL, USA, 2019.

117. Qu, Z.; Djordjevic, I.B.; Anderson, J. Two-Dimensional Constellation Shaping in Fiber-Optic Communications. Appl. Sci. 2019, 9, 1889. [CrossRef]

118. Millar, D.S.; Fehenberger, T.; Koike-Akino, T.; Kojima, K.; Parsons, K. Coded Modulation for Next-Generation Optical Communications. In Proceedings of the 2018 Optical Fiber Communications Conference and Exposition (OFC), San Diego, CA, USA, 11-15 March 2018.

119. Rajaram, A.; Jayakody, D.N.K.; Chen, B.; Dinis, R.; Affes, S. Modulation-based Simultaneous Wireless Information and Power Transfer. IEEE Commun. Lett. 2019, 24, 136-140. [CrossRef]

120. Liu, V.; Parks, A.; Talla, V.; Gollakota, S.; Wetherall, D.; Smith, J.R. Ambient backscatter: Wireless communication out of thin air. ACM SIGCOMM Comput. Commun. Rev. 2013, 43, 39-50. [CrossRef]

121. Van Huynh, N.; Hoang, D.T.; Lu, X.; Niyato, D.; Wang, P.; Kim, D.I. Ambient Backscatter Communications: A Contemporary Survey. IEEE Commun. Surv. Tutor. 2018, 20, 2889-2922. [CrossRef]

122. Darsena, D.; Gelli, G.; Verde, F. Cloud-Aided Cognitive Ambient Backscatter Wireless Sensor Networks. IEEE Access 2019, 7, 57399-57414. [CrossRef]

123. Zeb, S.; Abbas, Q.; Hassan, S.; Mahmood, A.; Zaidi, S.A.R.; Gidlund, M. NOMA Enhanced Backscatter Communication for Green IoT Networks. In Proceedings of the 2019 16th International Symposium on Wireless Communication Systems (ISWCS), Oulu, Finland, 27-30 August 2019. [CrossRef]

124. Bharadia, D.; Joshi, K.R.; Kotaru, M.; Katti, S. BackFi: High Throughput WiFi Backscatter. ACM SIGCOMM Comput. Commun. Rev. 2015, 45, 283-296. [CrossRef]

125. Wang, A.; Iyer, V.; Talla, V.; Smith, J.R.; Gollakota, S. FM Backscatter: Enabling Connected Cities and Smart Fabrics. In Proceedings of the 14th USENIX Symposium on Networked Systems Design and Implementatio, Boston, MA, USA, 27-29 March 2017.

126. Kellogg, B.; Talla, V.; Gollakota, S.; Smith, J.R. Passive Wi-Fi: Bringing Low Power to Wi-Fi Transmissions. In Proceedings of the 13th USENIX Symposium on Networked Systems Design and Implementation, Santa Clara, CA, USA, 16-18 March 2016; pp. 151-164.

127. Shirvanimoghaddam, M.; Mohammadi, M.S.; Abbas, R.; Minja, A.; Yue, C.; Matuz, B.; Han, G.; Lin, Z.; Liu, W.; Li, Y. Short block-length codes for ultra-reliable low latency communications. IEEE Commun. Mag. 2018, 57, 130-137. [CrossRef]

128. Niu, K.; Chen, K. CRC-Aided Decoding of Polar Codes. IEEE Commun. Lett. 2012, 16, 1668-1671. [CrossRef]

129. Alexiou, A. (Ed.) $5 G$ Wireless Technologies; Telecommunications, Institution of Engineering and Technology: London, UK, 2017.

130. Zheng, X.; Wang, G.; Zhao, Q. A Cache Placement Strategy with Energy Consumption Optimization in Information-Centric Networking. Future Internet 2019, 11, 64. [CrossRef]

131. Ji, J.; Zhu, K.; Wang, R.; Chen, B.; Dai, C. Energy Efficient Caching in Backhaul-Aware Cellular Networks with Dynamic Content Popularity. Wirel. Commun. Mob. Comput. 2018, 2018. [CrossRef]

132. Fan, C.; Zhang, T.; Zeng, Z.; Chen, Y. Energy Efficiency Analysis of Cache-Enabled Cellular Networks with Limited Backhaul. Wirel. Commun. Mob. Comput. 2018, 2018. [CrossRef]

133. Fan, C.; Zhang, T.; Zeng, Z.; Chen, Y. Backhaul Aware Energy Efficiency Analysis of Cache-Enabled Cellular Networks (Invited Paper). In Proceedings of the IEEE 87th Vehicular Technology Conference (VTC Spring), Porto, Portugal, 3-6 June 2018; pp. 1-5. [CrossRef] 
134. Paschos, G.S.; Gitzenis, S.; Tassiulas, L. The effect of caching in sustainability of large wireless networks. In Proceedings of the 10th International Symposium on Modeling and Optimization in Mobile, Ad Hoc and Wireless Networks (WiOpt)m, Paderborn, Germany, 14-18 May 2012; pp. 355-360.

135. Bhuvaneswari, P.; Nithyanandan, L. Improving Energy Efficiency in Backhaul of Lte-A Network With Base Station Cooperation. Procedia Comput. Sci. 2018, 143, 843-851. [CrossRef]

136. Qiu, L.; Cao, G. Cache increases the capacity of wireless networks. In Proceedings of the IEEE INFOCOM 2016-The 35th Annual IEEE International Conference on Computer Communications, San Francisco, CA, USA, 10-14 April 2016. [CrossRef]

137. Liu, D.; Chen, B.; Yang, C.; Molisch, A.F. Caching at the wireless edge: design aspects, challenges, and future directions. IEEE Commun. Mag. 2016, 54, 22-28. [CrossRef]

138. Niesen, U.; Shah, D.; Wornell, G. Caching in wireless networks. In Proceedings of the 2009 IEEE International Symposium on Information Theory, Seoul, South Korea, 28 June-3 July 2009; pp. 2111-2115. [CrossRef]

139. Cloud, Fog, and Edge Computing: 3 Differences That Matter-DZone Cloud. https://dzone.com/articles / cloud-vs-fog-vs-edge-computing-3-differences-that (accessed on 25 December 2019).

140. Berl, A.; Gelenbe, E.; Di Girolamo, M.; Giuliani, G.; Meer, H.; Dang, M.; Pentikousis, K. Energy-Efficient Cloud Computing. Comput. J. 2010, 53. [CrossRef]

141. Zhou, F.; Wu, Y.; Hu, R.; Wang, Y.; Wong, K.K. Energy-Efficient NOMA Enabled Heterogeneous Cloud Radio Access Networks. IEEE Netw. 2018. [CrossRef]

142. Peng, M.; Li, Y.; Jiang, J.; Li, J.; Wang, C. Heterogeneous Cloud Radio Access Networks: A New Perspective for Enhancing Spectral and Energy Efficiencies. IEEE Wirel. Commun. 2014, 21. [CrossRef]

143. Zhao, Z.; Peng, M.; Ding, Z.; Wang, W.; Poor, H.V. Cluster Content Caching: An Energy-Efficient Approach in Cloud Radio Access Networks. IEEE J. Sel. Areas Commun. 2016, 34. [CrossRef]

144. Ma, K.; Bagula, A.; Nyirenda, C.; Ajayi, O. An IoT-Based Fog Computing Model. Sensors 2019, $19,2783$. [CrossRef] [PubMed]

145. Basir, R.; Qaisar, S.; Ali, M.; Aldwairi, M.; Ashraf, M.I.; Mahmood, A.; Gidlund, M. Fog Computing Enabling Industrial Internet of Things: State-of-the-Art and Research Challenges. Sensors 2019, 19, 4807. [CrossRef]

146. Hu, P.; Dhelim, S.; Ning, H.; Qiu, T. Survey on fog computing: architecture, key technologies, applications and open issues. J. Netw. Comput. Appl. 2017, 98, 27-42. [CrossRef]

147. Li, Y.; Zhang, H.; Long, K.; Choi, S.; Nallanathan, A. Resource Allocation for Optimizing Energy Efficiency in NOMA-based Fog UAV Wireless Networks. IEEE Netw. 2019, 1-6. [CrossRef]

148. Wen, X.; Zhang, H.; Zhang, H.; Fang, F. Interference Pricing Resource Allocation and User-Subchannel Matching for NOMA Hierarchy Fog Networks. IEEE J. Sel. Top. Signal Process. 2019, 13, 467-479. [CrossRef]

149. Ai, Y.; Wang, L.; Jiao, B.; Chen, K. Exploiting NOMA into socially enabled computation offloading. In Proceedings of the 2017 9th International Conference on Wireless Communications and Signal Processing (WCSP), Nanjing, China, 11-13 October 2017. [CrossRef]

150. Liu, Y.; Xiong, K.; Zhang, Y.; Zhou, L.; Lin, F.; Liu, T. Multi-Objective Optimization of Fog Computing Assisted Wireless Powered Networks: Joint Energy and Time Minimization. Electronics 2019, 8, 137. [CrossRef]

151. Nath, S.; Gupta, H.; Chakraborty, S.; Ghosh, S. A Survey of Fog Computing and Communication: Current Researches and Future Directions. arXiv 2018, arXiv:1804.04365.

152. Wang, S.; Huang, X.; Liu, Y. CachinMobile: An energy-efficient users caching scheme for fog computing. 2016. [CrossRef]

153. Yang, Z.; Pan, C.; Hou, J.; Shikh-Bahaei, M. Efficient Resource Allocation for Mobile-Edge Computing Networks With NOMA: Completion Time and Energy Minimization. IEEE Trans. Commun. 2019, 67, 7771-7784. [CrossRef]

154. Jia, F.; Zhang, H.; Ji, H.; Li, X. Distributed Resource Allocation and Computation Offloading Scheme for Cognitive Mobile Edge Computing Networks with NOMA. In Proceedings of the IEEE/CIC International Conference on Communications in China (ICCC), Beijing, China, 16-18 August 2018; pp. 553-557. [CrossRef]

155. Quoc-Viet Pham and Fang Fang and Ha-Nguyen Vu and Mai Le and Zhiguo Ding and Long Bao Le and Won-Joo Hwang. A Survey of Multi-Access Edge Computing in 5G and Beyond: Fundamentals, Technology Integration, and State-of-the-Art. arXiv 2019, arXiv:1906.08452.

156. Nasir, A.; Hoang, T.; Duong, T.; Poor, H.V. UAV-Enabled Communication Using NOMA. IEEE Trans. Commun. 2019, 67, 5126-5138. [CrossRef] 
157. Yeom, J.S.; bin Kim, Y.; Jung, B.C. UAV-assisted cooperative downlink NOMA with virtual full-duplex operation. ICT Express 2019, 5, 240-244. [CrossRef]

158. Sohail, M.F.; Leow, C.Y.; Won, S. Energy-Efficient Non-Orthogonal Multiple Access for UAV Communication System. IEEE Trans. Veh. Technol. 2019, 68, 10834-10845. [CrossRef]

159. Gui, G.; Huang, H.; Song, Y.; Sari, H. Deep Learning for An Effective Non-Orthogonal Multiple Access Scheme. IEEE Trans. Veh. Technol. 2018. [CrossRef]

160. Zhang, S.; Liu, J.; Guo, H.; Qi, M.; Kato, N. Envisioning Device-to-Device Communications in 6G. arXiv 2019, arXiv:1912.05771.

161. Park, J.; Samarakoon, S.; Bennis, M.; Debbah, M. Wireless Network Intelligence at the Edge. Proc. IEEE 2019, 107, 2204-2239. [CrossRef]

162. Luo, J.; Tang, J.; So, D.K.C.; Chen, G.; Cumanan, K.; Chambers, J.A. A Deep Learning-Based Approach to Power Minimization in Multi-Carrier NOMA With SWIPT. IEEE Access 2019, 7, 17450-17460. [CrossRef]

163. Kosta, A.; Pappas, N.; Angelakis, V. Age of Information: A New Concept, Metric, and Tool. Found. Trends Netw. 2017, 12, 162-259. [CrossRef]

164. Maatouk, A.; Assaad, M.; Ephremides, A. Minimizing The Age of Information: NOMA or OMA? In Proceedings of the IEEE INFOCOM 2019-IEEE Conference on Computer Communications Workshops (INFOCOM WKSHPS), Paris, France, 29 April-2 May 2019.

165. Talak, R.; Karaman, S.; Modiano, E. Optimizing age of information in wireless networks with perfect channel state information. In Proceedings of the 16th International Symposium on Modeling and Optimization in Mobile, Ad Hoc, and Wireless Networks (WiOpt), Shanghai, China, 7-11 May 2018. [CrossRef]

166. Valehi, A.; Razi, A. Maximizing Energy Efficiency of Cognitive Wireless Sensor Networks with Constrained Age of Information. IEEE Trans. Cogn. Commun. Netw. 2017, 3, 643-654. [CrossRef]

167. Li, Z.; Gui, J. Energy-Efficient Resource Allocation With Hybrid TDMA-NOMA for Cellular-Enabled Machine-to-Machine Communications. IEEE Access 2019, 7, 105800-105815. [CrossRef]

168. Trestian, R.; Ormond, O.; Muntean, G.M. Game Theory-Based Network Selection: Solutions and Challenges. IEEE Commun. Surv. Tutor. 2012, 14, 1212-1231. [CrossRef]

169. Fettweis, G. The Tactile Internet: Applications and Challenges. IEEE Veh. Technol. Mag. 2014, 9, 64-70. [CrossRef]

170. Sharma, S.K.; Woungang, I.; Chatzinotas, S. Towards Tactile Internet in Beyond 5G Era: Recent Advances, Current Issues and Future Directions. arXiv 2019, arXiv:1908.07337.

171. Budhiraja, I.; Tyagi, S.; Tanwar, S.; Kumar, N.; Rodrigues, J.J.P.C. Tactile Internet for Smart Communities in 5G: An Insight for NOMA-Based Solutions. IEEE Trans. Ind. Inform. 2019, 15, 3104-3112. [CrossRef]

172. Budhiraja, I.; Tyagi, S.; Tanwar, S.; Kumar, N.; Rodrigues, J. DIYA: Tactile Internet Driven Delay Assessment NOMA-based Scheme for D2D Communication. IEEE Trans. Ind. Inform. 2019, 15, 1-11. [CrossRef]

173. Xu, J.; Ota, K.; Dong, M. Energy Efficient Hybrid Edge Caching Scheme for Tactile Internet in 5G. IEEE Trans. Green Commun. Netw. 2019, 3, 483-493. [CrossRef]

174. Ye, N.; Li, X.; Yu, H.; Wang, A.; Liu, W.; Hou, X. Deep Learning Aided Grant-Free NOMA Toward Reliable Low-Latency Access in Tactile Internet of Things. IEEE Trans. Ind. Inform. 2019, 15, 2995-3005. [CrossRef]

(C) 2020 by the authors. Licensee MDPI, Basel, Switzerland. This article is an open access article distributed under the terms and conditions of the Creative Commons Attribution (CC BY) license (http://creativecommons.org/licenses/by/4.0/). 